\title{
Possible and Necessary Winners of Partial Tournaments
}

\author{
Haris Aziz \\ Data61 and University of New South Wales \\ Australia
}

Markus Brill

Computer Science Department

Duke University, USA

Felix Fischer

Institut für Mathematik

Technische Universität Berlin, Germany

Paul Harrenstein

Computer Science Department

University of Oxford, UK

Jérôme Lang

LAMSADE

Université Paris-Dauphine, France

\author{
Hans Georg Seedig \\ Institut für Informatik \\ Technische Universität München, Germany
}

HARIS.AZIZ@NICTA.COM.AU

BRILL@CS.DUKE.EDU

FISCHERF@MATH.TU-BERLIN.DE

PAUL.HARRENSTEIN@CS.OX.AC.UK

LANG@LAMSADE.DAUPHINE.FR

SEEDIGH@IN.TUM.DE

\begin{abstract}
We study the problem of computing possible and necessary winners for partially specified weighted and unweighted tournaments. This problem arises naturally in elections with incompletely specified votes, partially completed sports competitions, and more generally in any scenario where the outcome of some pairwise comparisons is not yet fully known. We specifically consider a number of well-known solution concepts-including the uncovered set, Borda, ranked pairs, and maximin — and show that for most of them, possible and necessary winners can be identified in polynomial time. These positive algorithmic results stand in sharp contrast to earlier results concerning possible and necessary winners given partially specified preference profiles.
\end{abstract}

\section{Introduction}

Many multi-agent situations can be modeled and analyzed using weighted or unweighted tournaments. Prime examples are voting scenarios in which pairwise comparisons between alternatives are decided by majority rule and sports competitions that are organized as round-robin tournaments. Other application areas include webpage and journal ranking, biology, psychology, and AI. More generally, tournaments and solution concepts on tournaments are used as a mathematical tool for the analysis of all kinds of situations where a choice among a set of alternatives has to be made exclusively on the basis of pairwise comparisons.

When choosing from a tournament, relevant information may only be partly available. This could be because some preferences are yet to be elicited, some matches yet to be played, 
or certain comparisons yet to be made. In such cases, it is natural to speculate which are the potential and inevitable outcomes on the basis of the information already at hand.

For tournaments, a number of attractive solution concepts have been proposed (Brandt, Brill, \& Harrenstein, 2016; Laslier, 1997). Given any such solution concept $S$, we define possible winners of a partial tournament $G$ as alternatives that are selected by $S$ in some completion of $G$, and necessary winners are alternatives that are selected in all completions. By a completion we here understand a (complete) tournament extending $G$.

In this article we address the computational complexity of identifying the possible and necessary winners for a number of solution concepts whose winner determination problem for tournaments is tractable. We consider five of the most common solution concepts for tournaments-namely, Condorcet winners $(C O N D)$, Condorcet non-losers $(C N L)$, the Copeland set $(C O)$, the top cycle $(T C)$, and the uncovered set $(U C)$-and three common solutions for weighted tournaments - Borda $(B O)$, maximin $(M M)$, and ranked pairs $(R P)$. For each of these solution concepts, we consider the computational complexity of the following problems: deciding whether a given alternative is a possible winner (PW), deciding whether a given alternative is a necessary winner (NW), as well as deciding whether a given subset of alternatives equals the set of winners (the winning set) in some completion (PWS). These problems can be challenging, as even unweighted partial tournaments may allow for an exponential number of completions. Our results are encouraging, in the sense that most of the problems can be solved in polynomial time. Table 1 summarizes our findings.

Similar problems have been considered before. For Condorcet winners, voting trees and the top cycle, it has been shown that possible and necessary winners are computable in polynomial time (Konczak \& Lang, 2005; Lang et al., 2012). The same holds for the computation of possible Copeland winners, a problem that has been considered in the context of sports tournaments (Cook, Cunningham, Pulleyblank, \& Schrijver, 1998).

Another more specific setting is also frequently considered within the area of computational social choice and differs from our setting in a subtle but important way that is worth being pointed out. There, tournaments are assumed to arise from pairwise majority comparisons on the basis of a profile of individual voters' preferences. $^{1}$

Since a partial preference profile $R$ need not conclusively settle every majority comparison, it may give rise to a partial tournament only. There are then two natural ways to define possible and necessary winners for a partial preference profile $R$ and solution concept $S$ as illustrated in Figure 1. The first is to consider the completions of $R$ and the winners under $S$ in the corresponding tournaments. The second - covered by our more general setting - is to consider the completions of the partial tournament $G(R)$ corresponding to $R$ and the winners under $S$ in these. Since every tournament corresponding to a completion of $R$ is also a completion of $G(R)$ but not necessarily the other way round, the second definition

1. See, e.g., the work of Baumeister and Rothe (2010), Betzler and Dorn (2010), Konczak and Lang (2005), Walsh (2007), and Xia and Conitzer (2011) for the basic setting, Betzler, Hemmann, and Niedermeier (2009) for parameterized complexity results, Bachrach, Betzler, and Faliszewski (2010), Hazon, Aumann, Kraus, and Wooldridge (2012), and Kalech, Kraus, Kaminka, and Goldman (2011) for probabilistic settings, Chevaleyre, Lang, Maudet, and Monnot (2011) and Chevaleyre, Lang, Maudet, Monnot, and Xia (2012) for settings with a variable set of alternatives, Baumeister, Faliszewski, Lang, and Rothe (2012), Kalech et al. (2011), Lu and Boutilier (2011), Oren, Filmus, and Boutilier (2013), Filmus and Oren (2014) for settings with truncated ballots and Lu and Boutilier (2013) for multiwinner rules. 


\begin{tabular}{|c|c|c|c|c|}
\hline$S$ & $\mathrm{PW}_{S}$ & $\mathrm{NW}_{S}$ & & $\mathrm{PWS}_{S}$ \\
\hline$C O N D$ & in P (Konczak \& Lang, 2005) & in $\mathrm{P}$ & (Konczak \& Lang, 2005) & in $\mathrm{P} \quad$ (Thm. 1) \\
\hline$C N L$ & in $\mathrm{P}$ (Thm. 2) & in $\mathrm{P}$ & (Thm. 2) & in $\mathrm{P} \quad$ (Thm. 2) \\
\hline $\mathrm{CO}$ & in $\mathrm{P}(\text { Cook et al., } 1998)^{\mathrm{a}}$ & in $\mathrm{P}$ & $(\text { Thm. 3) })^{\mathrm{a}}$ & in $\mathrm{P}$ (Thm. 3) \\
\hline$T C$ & in $\mathrm{P}$ (Lang et al., 2012) ${ }^{\mathrm{a}}$ & in $\mathrm{P}$ & (Lang et al., 2012) & in $\mathrm{P}$ (Thm. 4) \\
\hline$U C$ & in $\mathrm{P} \quad$ (Thm. 5) & in $\mathrm{P}$ & (Thm. 6) & NP-C (Thm. 7) \\
\hline$B O$ & in $\mathrm{P}(\text { Thm. } 8)^{\mathrm{a}}$ & in $\mathrm{P}$ & (Thm. 10) & (Thm. 9) \\
\hline$M M$ & in $\mathrm{P}(\mathrm{Thm} .11)^{\mathrm{a}}$ & in $\mathrm{P}$ & (Thm. 13) & in $\mathrm{P}$ (Thm. 12) \\
\hline$R P$ & NP-C (Thm. 14) & coNP & (Thm. 15) & NP-C (Cor. 2) \\
\hline
\end{tabular}

a This P-time result contrasts with the intractability of the same problem for partial preference profiles (Lang et al., 2012; Xia \& Conitzer, 2011).

Table 1: Complexity of computing possible winners (PW) and necessary winners (NW) and of checking whether a given subset of alternatives is a possible winning set (PWS) under the following solution concepts: Condorcet winners $(C O N D)$, Condorcet non-losers $(C N L)$, Copeland $(C O)$, top cycle $(T C)$, uncovered set $(U C)$, Borda $(B O)$, maximin $(M M)$, and ranked pairs $(R P)$.

gives rise to a stronger notion of a possible winner and a weaker notion of a necessary winner. Interestingly, and in sharp contrast to our results, determining these stronger possible and weaker necessary winners is computationally hard for many voting rules (Lang et al., 2012; Xia \& Conitzer, 2011). This contrast has been foreshadowed by the work of Lang et al. (2012) and Pini, Rossi, Venable, and Walsh (2011), who compared these two ways of defining possible and necessary winners (both theoretically and experimentally) for three solution concepts: Condorcet winners, voting trees, and the top cycle.

In the context of this article, we do not assume that tournaments arise from majority comparisons in voting or from any other specific procedure. This approach has a number of advantages. Firstly, it matches the diversity of settings to which solution concepts on tournaments are applicable, which goes well beyond social choice and voting. For instance, our results also apply to a question commonly encountered in sports competitions, namely, which teams can still win the cup and which future results this depends on (Cook et al., 1998; Kern \& Paulusma, 2004; B. L. Schwartz, 1966). Secondly, (partial) tournaments provide an informationally sustainable way of representing the relevant aspects of many situations while maintaining a workable level of abstraction and conciseness. For instance, in the social choice setting described above, the partial tournament induced by a partial preference profile is a much more succinct piece of information, and discloses less information, than the preference profile itself. More generally, it gives a canonical way of extending tournament solutions to incomplete tournaments (a line that has been pursued by Brandt, Brill, \& Harrenstein, 2014). Finally, specific settings may impose restrictions on the feasible extensions of partial tournaments. The positive algorithmic results in this article can be used to efficiently approximate the sets of possible and necessary winners in such settings, where the corresponding problems may be intractable. The voting setting discussed above serves to illustrate this point.

We also point out that computing possible outcomes has been considered in other domains in social choice for example in matching and allocations (Aziz, Walsh, \& Xia, 2015; 


\begin{tabular}{lll}
1 & 1 & 1 \\
\hline$a$ & $c$ & \\
$b$ & $b$ & $a$ \\
$c$ & $a$ & $b$
\end{tabular}

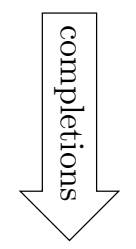

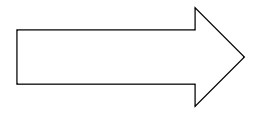
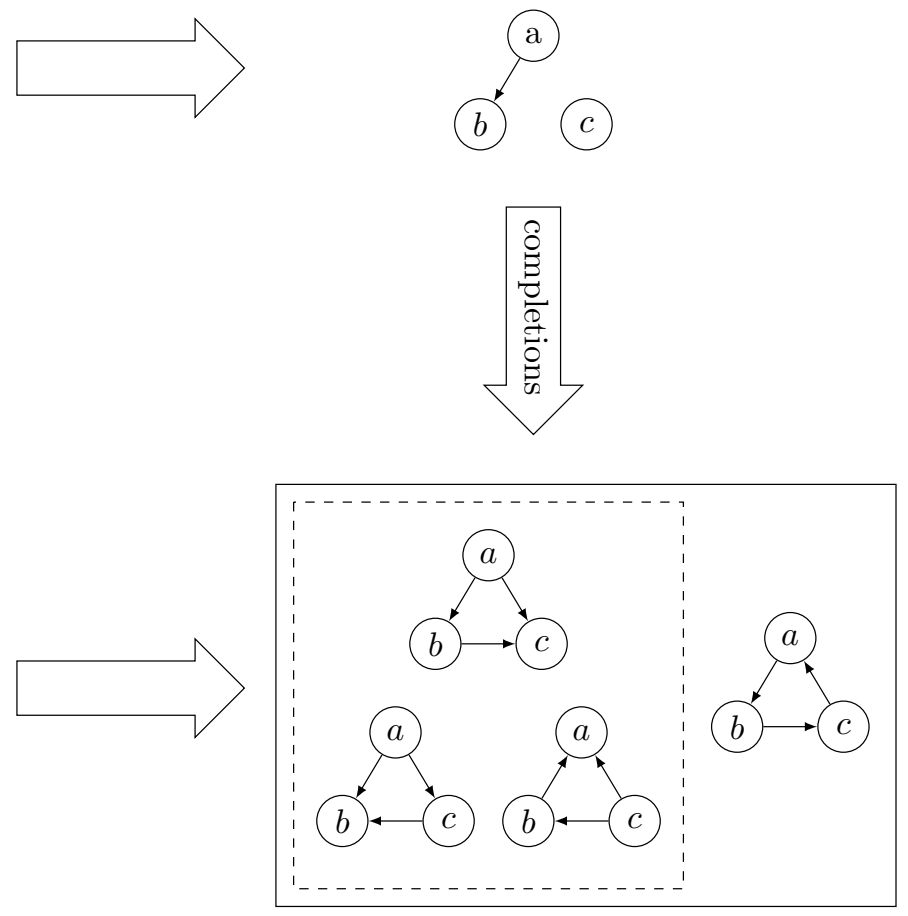

Figure 1: This non-commutative diagram illustrates the two approaches to possible and necessary winners of partial preference profiles for majoritarian social choice functions. First, the completions of the partial profile to full preference profiles are shown in the bottom left. The corresponding majority tournaments are in the dashed box on the bottom right. In this work, we start from the partial majority tournament on the top right which is induced by the partial preference profile. Then, we consider all possible completions to tournaments which are depicted in the solid box on the bottom right.

Rastegari, Condon, Immorlica, \& Leyton-Brown, 2013) and for knockout tournaments (Aziz et al., 2014; Vu, Altman, \& Shoham, 2009).

\section{Preliminaries}

A partial tournament is a pair $G=(V, E)$ where $V$ is a nonempty finite set of alternatives and $E \subseteq V \times V$ an asymmetric relation on $V$, i.e., $(y, x) \notin E$ whenever $(x, y) \in E$. If $(x, y) \in E$ we say that $x$ dominates $y$. A tournament $T$ is a partial tournament $(V, E)$ for which $E$ is also complete, i.e., either $(x, y) \in E$ or $(y, x) \in E$ for all distinct $x, y \in V$. We denote the set of all tournaments by $\mathscr{T}$.

Let $G=(V, E)$ be a partial tournament. Another partial tournament $G^{\prime}=\left(V^{\prime}, E^{\prime}\right)$ is called an extension of $G$, denoted $G \leq G^{\prime}$, if $V=V^{\prime}$ and $E \subseteq E^{\prime}$. If $E^{\prime}$ is complete, $G^{\prime}$ is called a completion of $G$. We write $[G]$ for the set of completions of $G$, i.e.,

$$
[G]=\{T \in \mathscr{T}: G \leq T\}
$$


We say an alternative $x \in V$ is dominated if $(y, x) \in E$ for some $y \in V$, and undominated otherwise. We define the dominion of $x$ in $G$ as $D_{G}^{+}(x)=\{y \in V:(x, y) \in E\}$, and the dominators of $x$ in $G$ as $D_{G}^{-}(x)=\{y \in V:(y, x) \in E\}$. For $X \subseteq V$, we let $D_{G}^{+}(X)=$ $\bigcup_{x \in X} D_{G}^{+}(x)$ and $D_{G}^{-}(X)=\bigcup_{x \in X} D_{G}^{-}(x)$. A nonempty subset $X \subseteq V$ of alternatives in a partial or complete tournament $(V, E)$ is dominant if every alternative in $X$ dominates every alternative outside $X$. For given $G=(V, E)$ and $X \subseteq V$, we further write $E^{X \rightarrow}$ for the set of edges obtained from $E$ by adding all missing edges from alternatives in $X$ to alternatives not in $X$, i.e.,

$$
E^{X \rightarrow}=E \cup\{(x, y) \in X \times V: y \notin X \text { and }(y, x) \notin E\}
$$

We use $E^{X \leftarrow}$ as an abbreviation for $E^{V \backslash X \rightarrow}$, and write $E^{x \rightarrow}, E^{x \leftarrow}, G^{X \rightarrow}$, and $G^{X \leftarrow}$ for

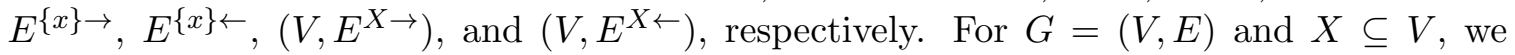
use $\left.E\right|_{X}$ and $\left.G\right|_{X}$ to denote the restriction $E \cap(X \times X)$ of $E$ to $X$ and the restriction $\left(X,\left.E\right|_{X}\right)$ of $G$ to $X$, respectively.

Let $n$ be a positive integer. A partial $n$-weighted tournament is a pair $G=(V, w)$ consisting of a finite set $V$ of alternatives and a weight function $w: V \times V \rightarrow\{0, \ldots, n\}$ such that for each pair $(x, y) \in V \times V$ with $x \neq y, w(x, y)+w(y, x) \leq n$. We say that $T=(V, w)$ is an $n$-weighted tournament if for all $x, y \in V$ with $x \neq y, w(x, y)+w(y, x)=n$. We call $T$ a (partial) weighted tournament if it is a (partial) $n$-weighted tournament for some $n \in \mathbb{N}$. The class of $n$-weighted tournaments is denoted by $\mathscr{T}_{n}$. Observe that with each partial 1-weighted tournament $(V, w)$ we can associate a partial tournament $(V, E)$ by setting $E=\{(x, y) \in V: w(x, y)=1\}$. Thus, (partial) $n$-weighted tournaments can be seen to generalize (partial) tournaments, and we may identify $\mathscr{T}_{1}$ with $\mathscr{T}$.

The notations $G \leq G^{\prime}$ and $[G]$ can be extended naturally to partial $n$-weighted tournaments $G=(V, w)$ and $G^{\prime}=\left(V^{\prime}, w^{\prime}\right)$ by letting $(V, w) \leq\left(V^{\prime}, w^{\prime}\right)$ if $V=V^{\prime}$ and $w(x, y) \leq w^{\prime}(x, y)$ for all $x, y \in V$, and $[G]=\left\{T \in \mathscr{T}_{n}: G \leq T\right\}$.

For given $G=(V, w)$ and $X \subseteq V$, we further define $w^{X \rightarrow}$ such that for all $x, y \in V$,

$$
w^{X \rightarrow}(x, y)= \begin{cases}n-w(y, x) & \text { if } x \in X \text { and } y \notin X \\ w(x, y) & \text { otherwise }\end{cases}
$$

and set $w^{X \leftarrow}=w^{V \backslash X \rightarrow}$. Moreover, $w^{x \rightarrow}, w^{x \leftarrow}, G^{X \rightarrow}$, and $G^{X \leftarrow}$ are defined in the obvious way.

We use the term solution concept for functions $S$ that associate with each tournament $T=(V, E)$, or with each weighted tournament $T=(V, w)$, a choice set $S(T) \subseteq V{ }^{2} \mathrm{~A}$ solution concept $S$ is called resolute if $|S(T)|=1$ for each tournament $T$. In this article we will consider the following solution concepts: Condorcet winners (COND), Condorcet nonlosers $(C N L)$, Copeland $(C O)$, top cycle $(T C)$, and uncovered set $(U C)$ for tournaments, and maximin $(M M)$, Borda $(B O)$, and ranked pairs $(R P)$ for weighted tournaments. Of these only ranked pairs is resolute. Formal definitions will be provided later in the article.

2. We avoid the otherwise natural term tournament solution as in its common definition it requires the choice set to be nonempty (Laslier, 1997). This would exclude COND. 


\section{Possible and Necessary Winners}

A solution concept selects a unique set of alternatives from each complete tournament. This holds in particular for the completions of a partial tournament. However, for each completion of a partial tournament, a solution concept may select another set of alternatives. A similar remark concerns weighted tournaments and their completions. For a given solution concept $S$, we can thus define the set of possible winners for a partial (weighted) tournament $G$ as the set of alternatives selected by $S$ from some completion of $G$, i.e., as

$$
P W_{S}(G)=\bigcup_{T \in[G]} S(T)
$$

Analogously, the set of necessary winners of $G$ is the set of alternatives selected by $S$ from every completion of $G$, i.e.,

$$
N W_{S}(G)=\bigcap_{T \in[G]} S(T)
$$

We furthermore write

$$
P W S_{S}(G)=\{S(T): T \in[G]\}
$$

for the possible winning sets, i.e., the set of sets of alternatives that $S$ selects for the different completions of $G$. For the sake of completeness, we also mention necessary winning sets. A set $X$ is a necessary winning set of a partial tournament $G$ if $X=S(T)$ for all $T \in[T]$. Accordingly, the conditions for a set to be a necessary winning set are very strong and are satisfied relatively seldom. Necessary winning sets can also straightforwardly be characterized by means of the sets of possible and necessary winners: $X$ is a necessary winning set if and only if $X=P W(G)=N W(G)$. This implies that for the solution concepts addressed in this article, computational results surrounding necessary winning sets follow as easy corollaries. ${ }^{3}$ We will not further consider necessary winning sets.

Note that $N W_{S}(G)$ may be empty even if $S$ selects a nonempty set of alternatives for each tournament $T \in[G]$, and that the number $\left|P W S_{S}(G)\right|$ of possible winning sets may be exponential in the number of alternatives of $G$.

We have the following lemmas, which relate to some useful structural properties of the sets of possible and necessary winners. The proofs are straightforward and therefore omitted.

Lemma 1. Let $S$ be a solution concept and $G$ and $G^{\prime}$ partial tournaments. Then,

(i) $G \leq G^{\prime}$ implies $P W_{S}\left(G^{\prime}\right) \subseteq P W_{S}(G)$, and

(ii) $G \leq G^{\prime}$ implies $N W_{S}(G) \subseteq N W_{S}\left(G^{\prime}\right)$.

We say that a solution concept $S$ refines another solution concept $S^{\prime}$, denoted $S \subseteq S^{\prime}$, if $S(G) \subseteq S^{\prime}(G)$ for all $G$. We find that the following monotonicity properties hold.

3. Given our results in Table 1 , the fact that $X$ is a necessary winning set if and only if $X=P W(G)=$ $N W(G)$ immediately implies that for all concepts apart from ranked pairs deciding whether a set is a necessary winning set can be achieved in polynomial time. Since ranked pairs is resolute, every set in $N W S_{R P}$ has to be a singleton $\{x\}$ and $\{x\} \in P W S_{R P}$ if and only if $x \in N W_{R P}$. Consequently, the problem of deciding whether a set $X$ is contained in $N W S_{R P}$ is coNP-complete. 
Lemma 2. Let $S$ and $S^{\prime}$ be solution concepts and $G$ and $G^{\prime}$ partial tournaments. Then,

(i) $S \subseteq S^{\prime}$ implies $P W_{S}(G) \subseteq P W_{S^{\prime}}(G)$, and

(ii) $S \subseteq S^{\prime}$ implies $N W_{S}(G) \subseteq N W_{S^{\prime}}(G)$.

The next lemma concerns the way in which the sets of possible and necessary winners can be defined in terms of one another.

Lemma 3. Let $S$ be a solution concept and $G$ a partial tournament. Then,

(i) $P W_{S}(G)=\bigcup_{G \leq G^{\prime}} N W_{S}\left(G^{\prime}\right)$, and

(ii) $N W_{S}(G)=\bigcap_{G \leq G^{\prime}} P W_{S}\left(G^{\prime}\right)$.

Observe that, while $S \subseteq S^{\prime}$ does not generally imply $P W S_{S}(G) \subseteq P W S_{S^{\prime}}(G)$, the following does hold:

if $S \subseteq S^{\prime}$ then for all $X \in P W S_{S}(G)$ there exists $X^{\prime} \in P W S_{S^{\prime}}(G)$ such that $X \subseteq X^{\prime}$.

Deciding membership in the sets $P W_{S}(G), N W_{S}(G)$, and $P W S_{S}(G)$ for a given solution concept $S$ and a partial (weighted) tournament $G$ are natural computational problems. Overloading notation, we refer to these problems as $\mathrm{PW}_{S}, \mathrm{NW}_{S}$, and $\mathrm{PWS}_{S}$, respectively.

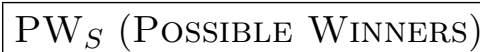

Input: $\quad$ A partial tournament $G=(V, E)$ or an $n$-weighted partial tournament $G=(V, w)$ along with the positive integer $n$; an alternative $x \in V$.

Output: 'Yes', if there exists a completion $T \in[G]$ such that $x \in S(T)$.

'No', otherwise.

$\mathrm{NW}_{S}$ (NECESSARY WiNNERS)

Input: A partial tournament $G=(V, E)$ or an $n$-weighted partial tournament $G=(V, w)$ along with the positive integer $n$; an alternative $x \in V$.

Output: 'Yes', if $x \in S(T)$ for all completions $T \in[G]$.

'No', otherwise.

$\mathrm{PWS}_{S}$ (Possible Winning Set)

Input: $\quad$ A partial tournament $G=(V, E)$ or an $n$-weighted partial tournament $G=(V, w)$ along with the positive integer $n$; a subset of alternatives $X \subseteq V$.

Output: 'Yes', if there exists a completion $T \in[G]$ such that $X=S(T)$. 'No', otherwise.

Note that if $\mathrm{PWS}_{S}$ can be decided in polynomial time, this only means that there is a polynomial-time algorithm that decides whether a given subset of alternatives is a possible 
winning set. Outputting the set $P W S_{S}$ of possible winning sets may be much more difficult, because $P W S_{S}$ may be of exponential size. ${ }^{4}$

For irresolute solution concepts, $\mathrm{PWS}_{S}$ may appear a more complex problem than $\mathrm{PW}_{S}$. We are, however, not aware of a generic polynomial-time reduction from $\mathrm{PW}_{S}$ to $\mathrm{PWS}_{S}$. The relationship between all of these problems may also be of interest for the "classic" possible winner setting with partial preference profiles.

For complete tournaments $T$ we have $[T]=\{T\}$ and thus $P W_{S}(T)=N W_{S}(T)=S(T)$ and $P W S_{S}(T)=\{S(T)\}$. As a consequence, for solution concepts $S$ with an NP-hard winner determination problem-like Banks, Slater, and the tournament equilibrium setthe problems $\mathrm{PW}_{S}, \mathrm{NW}_{S}$, and $\mathrm{PWS}_{S}$ are NP-hard as well. ${ }^{5}$ We therefore restrict our attention to solution concepts for which winners can be computed in polynomial time.

\section{Unweighted Tournament Solutions}

In this section, we consider the following well-known solution concepts for unweighted tournaments: Condorcet winners, Condorcet non-losers, the Copeland set, the top cycle, and the uncovered set. We will use the partial tournament depicted in Figure 2(i) as a running example.

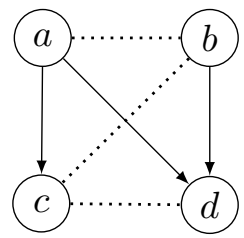

(i) Partial tournament $G$

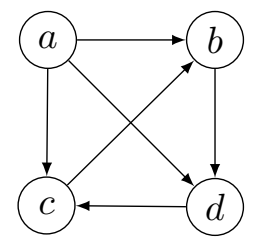

(ii) Completion $T_{1}$

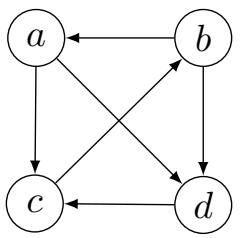

(iii) Completion $T_{2}$

Figure 2: Example of a partial unweighted tournament $G$ and possible completions $T_{1}$ and $T_{2}$. Initially, the (dotted) edges between the pairs $\{a, b\},\{b, c\}$, and $\{c, d\}$ are not yet specified.

\subsection{Condorcet Winners and Condorcet Non-losers}

Condorcet winners and Condorcet non-losers are fundamental solution concepts and will provide a nice warm-up. An alternative $x \in V$ is a Condorcet winner of a complete tournament $T=(V, E)$ if it dominates all other alternatives, i.e., if $(x, y) \in E$ for all $y \in V \backslash\{x\}$. The set of Condorcet winners of tournament $T$ will be denoted by $C O N D(T)$; obviously this set is always either a singleton or empty. An alternative $x$ is a Condorcet loser in $T$ if it is dominated by every other alternative, i.e., if $(y, x) \in E$ for all $y \in V \backslash\{x\}$. Consequently, $x$ is a Condorcet non-loser in $T=(V, E)$ if $x$ is not a Condorcet loser in $T$ or $V=\{x\}$.

The set of Condorcet non-losers of a tournament $T$ will be denoted by $C N L(T)$; obviously this set has always cardinality $|V|$ or $|V|-1$.

4. For instance, if $G_{\emptyset}=(V, \emptyset)$ then $P W S_{T C}\left(G_{\emptyset}\right)=\{X \subseteq V:|X| \neq 2\}$, even though $\mathrm{PWS}_{T C}$ is in $\mathrm{P}$ (Theorem 4).

5. This does not exclude the possibility that computing some (arbitrary) possible winner or possible winning set for some of these solution concepts could be done in polynomial time. 
Let $G=(V, E)$ be a partial tournament. If some alternative $x$ is dominant in $G$, then $x$ will obviously be the Condorcet winner in all completions of $G$. On the other hand, if for some $y \in V \backslash\{x\}$ it is not the case that $(x, y) \in E$, there is some completion of $G$ in which $x$ is not a Condorcet winner. Hence,

$$
x \in N W_{C O N D}(G) \text { if and only if }(x, y) \in E \text { for all } y \in V \backslash\{x\}
$$

and

$$
x \in P W_{C O N D}(G) \text { if and only if }(y, x) \in E \text { for no } y \in V \backslash\{x\} .
$$

Obviously, the criteria on the right-hand side of the equivalences can be checked in polynomial time.

We now turn to the problem $\mathrm{PWS}_{C O N D}$. Each of the sets in $P W S_{C O N D}(G)$ is either a singleton or the empty set, and determining membership for a singleton is obviously tractable. Checking whether $\emptyset \in P W S_{C O N D}(G)$ is not quite that simple. The following result gives an exact characterization of $P W S_{C O N D}(G)$, which is interesting in its own right.

Lemma 4. Let $U$ be the set of undominated alternatives of a partial tournament $G=(V, E)$. Then,

- for every alternative $x \in V,\{x\} \in P W S_{C O N D}(G)$ if and only if $x \in U$;

- $\emptyset \notin P W S_{C O N D}(G)$ if and only if $1 \leq|U| \leq 2$ and $U$ is dominant.

Proof. Since a complete tournament has either one Condorcet winner or none, any set in $P W S_{C O N D}(G)$ has cardinality 0 or 1 . Clearly, $\{x\} \in P W S_{C O N D}(G)$ if and only if $x \in U$. It remains to be shown that $P W S_{C O N D}(G)$ contains $\emptyset$ if and only if $U=\emptyset$, or $|U| \geq 3$, or $1 \leq|U| \leq 2$ and $U$ is not dominant.

If $U=\emptyset, C O N D(T)=\emptyset$ for every $T \in[G]$. It follows that $\emptyset \in P W S_{C O N D}(G)$. If $|U| \geq 3$, consider a directed cycle $C \subseteq U \times U$ that visits every alternative in $U .{ }^{6}$ Then, the set of undominated alternatives in $G^{\prime}=(V, E \cup C)$ is empty. It again follows that $\emptyset \in P W S_{C O N D}(G)$.

If $U=\{x\}$ and $x$ is dominant, then $x$ is a Condorcet winner in every $T \in[G]$. Therefore, $\emptyset \notin P W S_{C O N D}(G)$.

If $U=\{x\}$ and $\{x\}$ is not dominant, then $(x, y) \notin E$ for some $y \neq x$. Consider a completion of $G$ containing $(y, x)$. In this completion, the set of undominated alternatives is empty. It follows that $\emptyset \in P W S_{C O N D}(G)$.

If $U=\{x, y\}$ and $\{x, y\}$ is dominant, then for every $T \in[G]$, either $(x, y) \in T$ and $x$ is a Condorcet winner in $T$, or $(y, x) \in T$ and $y$ is a Condorcet winner in $T$. It follows that $\emptyset \notin P W S_{C O N D}(G)$.

Finally, if $U=\{x, y\}$ and $\{x, y\}$ is not dominant, then for some $z \neq x, y$ we have $(x, z) \notin E$ or $(y, z) \notin E$. Without loss of generality, assume $(x, z) \notin E$. Consider a completion of $G$ containing $(z, x)$ and $(x, y)$. Such a completion exists, because $(x, z) \notin E$, and $(y, x) \notin E$ (since $x \in U)$. In this completion, the set of undominated alternatives is empty. It follows that $\emptyset \in P W S_{C O N D}(G)$.

6. The cycle $C$ is not a subgraph of $G$. In fact, $\left.G\right|_{U}$ does not contain any edges. 
We are now in a position to prove the following theorem.

Theorem 1. $\mathrm{PW}_{C O N D}, \mathrm{NW}_{C O N D}$, and $\mathrm{PWS}_{C O N D}$ can all be solved in polynomial time.

The results for $\mathrm{PW}_{C O N D}$ and $\mathrm{NW}_{C O N D}$ also follow from Corollary 2 of Konczak and Lang (2005).

We further note that Theorem 1 is a corollary of corresponding results for maximin in Section 5.2. The reason is that a Condorcet winner is the maximin winner of a 1weighted tournament, and a tournament does not admit a Condorcet winner if and only if all alternatives are maximin winners.

We conclude this section by observing that the problems $\mathrm{PW}_{C N L}, \mathrm{NW}_{C N L}$, and $\mathrm{PWS}_{C N L}$ are reducible to $\mathrm{NW}_{C O N D}, \mathrm{PW}_{C O N D}$, and $\mathrm{PWS}_{C O N D}$, respectively. It can straightforwardly be checked that for all partial tournaments $G=(V, E)$ with $|V|>1$ and all $X \subseteq V$,

$$
X \in P W S_{C N L}(G) \text { if and only if } V \backslash X \in P W S_{C O N D}\left(G^{-1}\right),
$$

where $G^{-1}=\left(V, E^{-1}\right)$ is $G$ with all of its set edges inverted, i.e., $E^{-1}=\{(x, y):(y, x) \in E\}$. It also follows that,

$$
\begin{aligned}
& P W_{C N L}(G)=V \backslash N W_{C O N D}\left(G^{-1}\right), \text { and } \\
& N W_{C N L}(G)=V \backslash P W_{C O N D}\left(G^{-1}\right) .
\end{aligned}
$$

Since the complement of a set can be computed in polynomial time and edges can be reversed in polynomial time as well, we obtain the following result as a corollary of Theorem 1.

Theorem 2. $\mathrm{PW}_{C N L}, \mathrm{NW}_{C N L}$, and $\mathrm{PWS}_{C N L}$ can all be solved in polynomial time.

As an example, consider the partial tournament $G$ depicted in Figure $2(i)$ in which there is no dominating alternative while the set of undominated alternatives in $G$ is $U=\{a, b\}$. Therefore,

$$
\begin{aligned}
& P W_{C O N D}(G)=\{a, b\} \text { and } \\
& N W_{C O N D}(G)=\emptyset .
\end{aligned}
$$

For $P W S_{C O N D}(G)$, note that the set $U$ is not dominant because $(b, c) \notin E$. By Lemma 4 , this gives

$$
P W S_{C O N D}(G)=\{\{a\},\{b\}, \emptyset\} .
$$

For Condorcet non-losers, we observe that $G^{-1}=\left(V, E^{-1}\right)$ with $E^{-1}=\{(c, a),(d, a),(d, b)\}$. Now, $P W_{C O N D}\left(G^{-1}\right)=\{c, d\}, \quad N W_{C O N D}\left(G^{-1}\right)=\emptyset$, and (from Lemma 4) $P W S_{C O N D}\left(G^{-1}\right)=\{\{c\},\{d\}, \emptyset\}$. Therefore,

$$
\begin{aligned}
P W_{C N L}(G) & =\{a, b, c, d\} \\
N W_{C N L}(G) & =\{a, b\}, \text { and } \\
P W_{C N L}(G) & =\{\{a, b, d\},\{a, b, c\},\{a, b, c, d\}\} .
\end{aligned}
$$




\subsection{Copeland}

Copeland's solution selects alternatives based on the number of other alternatives they dominate. Define the Copeland score of an alternative $x$ in tournament $T=(V, E)$ as

$$
s_{C O}(x, T)=\left|D_{T}^{+}(x)\right| .
$$

The set $C O(T)$ then consists of all alternatives that have maximal Copeland score.

For an illustrative example, consider again the partial tournament $G$ shown in Figure $2(i)$. In completions of $G$ where $a$ (respectively $b$ ) is a Condorcet winner, $a$ (respectively $b$ ) is the sole Copeland winner as in the completion shown in Figure 2(ii). The only two completions in which neither $a$ nor $b$ is a Condorcet winner are

$$
\{(a, c),(a, d),(b, a),(b, d),(c, b),(c, d)\}
$$

where the set of Copeland winners is $\{a, b, c\}$, and

$$
\{(a, c),(a, d),(b, a),(b, d),(c, b),(d, c)\}
$$

also depicted in Figure 2(iii), where the set of Copeland winners is $\{a, b\}$. Therefore,

$$
\begin{aligned}
P W_{C O}(G) & =\{a, b, c\}, \\
N W_{C O}(G) & =\emptyset, \text { and } \\
P W S_{C O}(G) & =\{\{a\},\{b\},\{a, b\},\{a, b, c\}\} .
\end{aligned}
$$

Since Copeland scores coincide with Borda scores in the case of 1-weighted tournaments, the following is a direct corollary of the results in Section 5.1. ${ }^{7}$

Theorem 3. $\mathrm{PW}_{C O}, \mathrm{NW}_{C O}$, and $\mathrm{PWS}_{C O}$ can all be solved in polynomial time.

From $\mathrm{PWS}_{C O}$ being solvable in polynomial time, we get the following corollary, which may be of independent interest to graph theorists.

Corollary 1. There exists a polynomial-time algorithm to check whether a partial tournament admits a regular completion, i.e., a completion in which every alternative has the same out-degree.

To see this, merely observe that a completion $T=(V, E)$ of a partial tournament is regular if and only if $C O(T)=V$.

\subsection{Top Cycle}

The top cycle of a tournament $T=(V, E)$, denoted by $T C(T)$, is the unique minimal dominant subset of $V$.

Lang et al. have shown that possible and necessary winners for $T C$ can be computed efficiently by greedy algorithms (Lang et al., 2012, Corollaries 1 and 2). Still, we give the following characterization that will prove useful when we come to consider the possible

7. $\mathrm{PW}_{C O}$ can alternatively be solved via a polynomial-time reduction to maximum network flow (Cook et al., 1998, p. 51). 
winning sets under $T C$. An alternative is a possible $T C$-winner if and only if it can reach every other alternative via existing or unspecified edges. Formally, given a partial tournament $G=(V, E)$, an alternative $x \in V$ is in $P W_{T C}(G)$ if and only if for every other alternative $y \in V$, there exists a path $x_{0}, x_{1}, x_{2}, \ldots, x_{k}$ with $x=x_{0}, y=x_{k}$, and such that $\left(x_{i+1}, x_{i}\right) \notin E$ for all $i \in\{0, \ldots, k-1\}$. We call such a path a possible path. If a possible path from $x$ to $y$ exists, we denote that by $x \rightsquigarrow y$.

Observe that for any pair $a$ and $b$ of alternatives in a partial tournament $G=(V, E)$, if there is no possible path from $a$ to $b$, then $(b, a) \in E$. The set of alternatives that can reach every other alternative via a possible path in a partial tournament $G=(V, E)$ is also known as the Good set (Good, 1971) and is denoted by $G O(G){ }^{8}$ It follows from the above that $G O(G)$ is polynomial-time computable. Moreover, we have the following lemma.

Lemma 5. Let $G=(V, E)$ be a partial tournament with $|V| \geq 3, G O(G)=V$, and $x$ and $y$ alternatives such that $(x, y),(y, x) \notin E$. Let further $G^{x \rightarrow y}=(V, E \cup\{(x, y)\})$ and $G^{y \rightarrow x}=(V, E \cup\{(y, x)\})$. Then, $G O\left(G^{x \rightarrow y}\right)=V$ or $G O\left(G^{y \rightarrow x}\right)=V$.

Proof. Assume for contradiction that both $G O\left(G^{x \rightarrow y}\right)$ and $G O\left(G^{y \rightarrow x}\right)$ are strict subsets of $V$. Clearly, we have $x \in G O\left(G^{x \rightarrow y}\right)$ and $y \in G O\left(G^{y \rightarrow x}\right)$. Moreover, we claim that $x \notin$ $G O\left(G^{y \rightarrow x}\right)$ and $y \notin G O\left(G^{x \rightarrow y}\right)$. To see why $x \notin G O\left(G^{y \rightarrow x}\right)$ holds, assume for contradiction that $x \in G O\left(G^{y \rightarrow x}\right)$. Then, there is a possible path from $x$ to $y$ in $G^{y \rightarrow x}$. This path can be used to "replace" the edge $(x, y)$ (which is available for possible paths in $G$, but not in $\left.G^{y \rightarrow x}\right)$. Therefore, there is a possible path between two alternatives in $G^{y \rightarrow x}$ whenever there is one in $G$. Since $G O(G)=V$, we have $G O\left(G^{y \rightarrow x}\right)=V$ as well, contradicting our assumption. An analogous argument shows that $y \notin G O\left(G^{x \rightarrow y}\right)$.

Having established that $x \in G O\left(G^{x \rightarrow y}\right) \backslash G O\left(G^{y \rightarrow x}\right)$ and $y \in G O\left(G^{y \rightarrow x}\right) \backslash G O\left(G^{x \rightarrow y}\right)$, we know that there is no possible path from $x$ to $y$ in $G^{y \rightarrow x}$, and neither is there a possible path from $y$ to $x$ in $G^{x \rightarrow y}$. Now consider some $z \in V \backslash\{x, y\}$. We have that either

(i) $(x, z) \in E$ and $(y, z) \in E$, or

(ii) $(z, x) \in E$ and $(z, y) \in E$,

as otherwise there would be either a possible path from $x$ to $y$ in $G^{y \rightarrow x}$ or a possible path from $y$ to $x$ in $G^{x \rightarrow y}$.

If $(i)$, recall that we have assumed that $G O(G)=V$. Hence, in $G$ there are possible paths $z \rightsquigarrow x$ and $z \rightsquigarrow y$. Observe that we may assume that either the possible path from $z$ to $x$ does not contain $(y, x)$, or the possible path from $z$ to $y$ does not contain $(x, y)$. In the former case, $y, z \rightsquigarrow x$ is a possible path in $G^{x \rightarrow y}$. In the latter case, $x, z \rightsquigarrow y$ is a possible path in $G^{y \rightarrow x}$. Thus, either case yields a contradiction.

If $(i i), G O(G)=V$ implies that there are possible paths $x \rightsquigarrow z$ and $y \rightsquigarrow z$ in $G$, and we may assume that either the possible path from $x$ to $z$ does not contain $(y, x)$ or the possible path from $y$ to $z$ that does not contain $(x, y)$. In the former case, there is a possible path $x \rightsquigarrow z, y$ in $G^{x \rightarrow y}$. In the latter case, there is a possible path $y \rightsquigarrow z, x$ in $G^{y \rightarrow x}$. Again, either case leads to a contradiction. This concludes the proof.

8. Equivalently, the Good set of a partial tournament $G=(V, E)$ is the unique minimal dominant subset of $V$. The Good set is also known as the Smith set (Smith, 1973) and GETCHA (T. Schwartz, 1986). 
We are now ready to show that $\mathrm{PWS}_{T C}$ can be solved efficiently. Note that we not only have to check that there exists a completion such that the set in question is dominating, but also that there is no smaller dominating set.

Theorem 4. $\mathrm{PWS}_{T C}$ can be solved in polynomial time.

Proof. Let the set under consideration be $X$. The set $X$ cannot be empty as $T C(T) \neq \emptyset$ for every $T \in[G]$. If $|X|=1$, then the problem $\mathrm{PWS}_{T C}$ is equivalent to $\mathrm{PW}_{C O N D}$. If $|X|=2$, then the answer is already 'no' because the top cycle is never of size two. We may therefore assume that $|X| \geq 3$.

Consider the graph $G^{X \rightarrow}$. If $X$ does not dominate $V \backslash X$ in $G^{X \rightarrow}$, then $X \notin P W S_{T C}(G)$ because an alternative in $V \backslash X$ beats an alternative in $X$. Therefore, we now need to check whether $X \in P W S_{T C}\left(\left.G\right|_{X}\right)$, i.e., whether $X$ is a possible top cycle set in the partial tournament $G$ restricted to $X$. In essence, the problem $\mathrm{PWS}_{T C}$ is reduced to the restricted problem PWS $\mathrm{PWC}_{T C}$ for the set of all alternatives.

We prove that $V \in P W S_{T C}(G)$ if and only if $G O(G)=V$. Obviously, if $V \neq G O(G)$ then $V \notin P W S_{T C}(G)$. For the other direction, we start with a partial tournament $G=$ $(V, E)$ with $G O(G)=V$. By iteratively applying Lemma 5, new edges can successively be added to $G$ while maintaining $G O(G)=V$ until $G$ is a tournament.

As an example, we again consider the partial tournament $G$ depicted in Figure $2(i)$, for which we show that

$$
\begin{aligned}
P W_{T C}(G) & =\{a, b, c, d\} \\
N W_{T C}(G) & =\emptyset, \text { and } \\
P W S_{T C}(G) & =\{\{a\},\{b\},\{a, b, c\},\{a, b, c, d\}\} .
\end{aligned}
$$

The result for $P W_{T C}(G)$ is witnessed by the completion shown in Figure 2(iii) where every alternative is in the top cycle. For $N W_{T C}(G)$, the statement follows from the observation that for every alternative, there exists a completion in which another alternative is a Condorcet winner. Regarding $P W S_{T C}(G)$, we consider each subset separately. Since $P W S_{C O N D} \subseteq P W S_{T C}$, we get that $\{a\}$ and $\{b\}$ are in $P W S_{T C}(G)$. For $\{a, b, c\}$, we apply the result shown in the second paragraph of the proof of Theorem 4: $a, b, c$ are undominated by $d$, and the Good set of $\left.G\right|_{\{a, b, c\}}$ is $\{a, b, c\}$. Likewise, the Good set of $G$ is $\{a, b, c, d\}$. It remains to be shown that the other subsets of size three are not in $P W S_{T C}(G)$. To this end, note that the Good set of $\left.G\right|_{\{a, b, d\}}$ is only $\{a, b\}$ and that neither $\{a, c, d\}$ nor $\{b, c, d\}$ is undominated in $G$.

\subsection{Uncovered Set}

Given a tournament $T=(V, E)$, an alternative $x \in V$ is said to cover another alternative $y \in V$ if $D_{T}^{+}(y) \subseteq D_{T}^{+}(x)$, i.e., if every alternative dominated by $y$ is also dominated by $x$. The uncovered set of $T$, denoted $U C(T)$, then is the set of alternatives that are not covered by any other alternative. A useful alternative characterization of the uncovered set is via the two-step principle: an alternative is in the uncovered set if and only if it can reach every other alternative in at most two steps. ${ }^{9}$ Formally, $x \in U C(T)$ if and only if for all

9. In graph theory, vertices satisfying this property are often called kings. 
$y \in V \backslash\{x\}$, either $(x, y) \in E$ or there is some $z \in V$ with $(x, z),(z, y) \in E$. We denote the two-step dominion $D_{E}^{+}\left(D_{E}^{+}(x)\right)$ of an alternative $x$ by $D_{E}^{++}(x)$.

We first consider $\mathrm{PW}_{U C}$, for which we check for each alternative whether it can be reinforced to reach every other alternative in at most two steps.

Theorem 5. $\mathrm{PW}_{U C}$ can be solved in polynomial time.

Proof. For a given partial tournament $G=(V, E)$ and an alternative $x \in V$, we check whether $x$ is in $U C(T)$ for some completion $T \in[G]$.

Consider the graph $G^{\prime}=\left(V, E^{\prime \prime}\right)$ where $E^{\prime \prime}$ is derived from $E$ as follows. First, we let $D^{+}(x)$ grow as much as possible by letting $E^{\prime}=E^{x \rightarrow}$. Then, we do the same for its

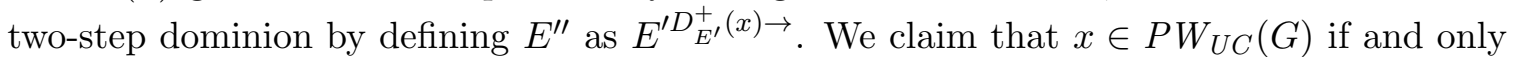
if $V=\{x\} \cup D_{E^{\prime \prime}}^{+}(x) \cup D_{E^{\prime \prime}}^{++}(x)$.

$(\Rightarrow)$ First, let $x \in P W_{U C}(G)$. By definition, there is a completion $\left(V, E^{*}\right)$ such that for all $y \in V \backslash\{x\}$ we have $y \in D_{E^{*}}^{+}(x) \cup D_{E^{*}}^{++}(x)$. But from the definition of $E^{\prime \prime}$, it follows that $D_{E^{*}}^{+}(x) \subseteq D_{E^{\prime \prime}}^{+}(c)$ and $D_{E^{*}}^{++}(x) \subseteq D_{E^{\prime \prime}}^{++}(x)$. Consequently, $y$ is also in $D_{E^{\prime \prime}}^{+}(x) \cup D_{E^{\prime \prime}}^{++}(x)$.

$(\Leftarrow)$ For the other direction, let $y \in V \backslash\{x\}, y \in D_{E^{\prime \prime}}^{+}(x) \cup D_{E^{\prime \prime}}^{++}(x)$. In any completion $T$ of $G^{\prime}, x$ is trivially in $U C(T)$, implying that $x \in P W_{U C}(G)$.

A similar argument yields the following.

Theorem 6. $\mathrm{NW}_{U C}$ can be solved in polynomial time.

Proof. For a given partial tournament $G=(V, E)$ and an alternative $x \in V$, we check whether $x$ is in $U C(T)$ for all completions $T \in[G]$.

Consider the graph $G^{\prime}=\left(V, E^{\prime \prime}\right)$ with $E^{\prime \prime}$ defined as follows. First, let $E^{\prime}=E^{x \leftarrow}$. Then, expand it to $E^{\prime \prime}=E^{\prime D_{E^{\prime}}^{-}(x) \leftarrow}$. Intuitively, this makes it as hard as possible for $x$ to beat alternatives outside of its dominion in two steps. We claim that $x \in N W_{U C}(G)$ if and only if $V=\{x\} \cup D_{E^{\prime \prime}}^{+}(x) \cup D_{E^{\prime \prime}}^{++}(x)$ or equivalently, if and only if for all $y \neq x$ there is a path of length one or two from $x$ to $y$ in $G$.

$(\Rightarrow)$ First, let $x \in N W_{U C}(G)$. Assume for contradiction that there exists a $y \in V \backslash\{x\}$ such that $y \notin D_{E^{\prime \prime}}^{+}(x) \cup D_{E^{\prime \prime}}^{++}(x)$. Then, in any completion $\left(V, E^{*}\right)$ of $G^{\prime}, x$ cannot reach $y$ in two steps and consequently $x \notin U C\left(V, E^{*}\right)$, a contradiction.

$(\Leftarrow)$ Now, let $V \backslash\{x\}=D_{E^{\prime \prime}}^{+}(x) \cup D_{E^{\prime \prime}}^{++}(x)$. In any completion $\left(V, E^{*}\right)$ of $G$, we have $D_{E^{\prime \prime}}^{+}(x) \subseteq D_{E^{*}}^{+}(x)$ and $D_{E^{\prime \prime}}^{++}(x) \subseteq D_{E^{*}}^{++}(x)$. Consequently, $x \in U C\left(V, E^{*}\right)$ and $x \in N W_{U C}(G)$.

As it can be checked in polynomial time whether $V=\{x\} \cup D_{E^{\prime \prime}}^{+}(x) \cup D_{E^{\prime \prime}}^{++}(x)$, this completes the proof.

Consider the partial tournament $G$ from Figure $3(i)$ as an example. It can be checked that $N W_{U C}(G)=\emptyset .{ }^{10}$ For $P W_{U C}$, we consider each alternative separately. For $a$, we have $E^{\prime}=E^{a \rightarrow}=\{(a, b),(a, c),(a, d),(b, d)\}$, and $E^{\prime \prime}=E^{\prime}$, therefore $D_{E^{\prime \prime}}^{+}(a)=\{b, c, d\}$ and $a \in P W_{U C}(G)$. Likewise, $b \in P W_{U C}(G)$. Now, for $c$, we have $E^{\prime}=\{(a, c),(a, d),(b, d),(c, b),(c, d)\}$ and $E^{\prime \prime}=\{(a, c),(a, d),(b, d),(c, b),(c, d),(b, a)\}$, see also Figure $3(i i)$. This gives us $D_{E^{\prime \prime}}^{+}(c)=\{b, d\}$ and $D_{E^{\prime \prime}}^{++}(c)=\{a\}$, and therefore, $c \in P W_{U C}(G)$. Lastly, for $d$, we have $E^{\prime}=\{(a, c),(a, d),(b, d),(d, c)\}$ and $E^{\prime \prime}=$

10. This is also a consequence of $N W_{T C}(G)=\emptyset$ (Section 4.3) and $N W_{U C} \subseteq N W_{T C}$ (Lemma 2). 


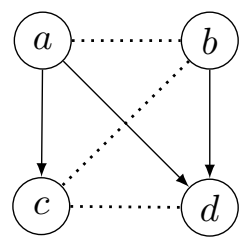

(i)

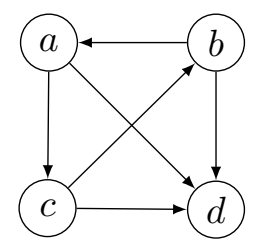

(ii)

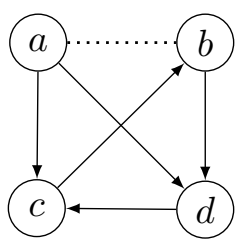

(iii)

Figure 3: A partial unweighted tournament $G$ and possible extensions. In the center, the alternative $c$ and its dominion is maximally reinforced resulting in $c$ reaching every other alternative in at most two steps. Therefore, $c \in P W_{U C}(G)$. On the right, the same is done for alternative $d$ that cannot reach $a$ in two steps and is therefore not contained in $P W_{U C}(G)$.

$\{(a, c),(a, d),(b, d),(d, c),(c, b)\}$ as depicted in Figure $3(i i i)$. This gives us $D_{E^{\prime \prime}}^{+}(d)=\{c\}$ and $D_{E^{\prime \prime}}^{++}(d)=\{b\}$, implying that $d \notin P W_{U C}(G)$. In summary,

$$
\begin{aligned}
P W_{U C}[G] & =\{a, b, c\}, \\
N W_{U C}(G) & =\emptyset, \text { and } \\
P W S_{U C}(G) & =\{\{a\},\{b\},\{a, b, c\}\},
\end{aligned}
$$

where $P W S_{U C}(G)$ is obtained by an ad hoc argument.

For all solution concepts considered so far-Condorcet winners, Condorcet non-losers, Copeland, and the top cycle-PW and PWS have the same complexity. One might wonder whether a result like this holds more generally, and whether there could be a polynomialtime reduction from PWS to PW. In the following we find that this is not the case, unless $\mathrm{P}=\mathrm{NP}$, and show that $\mathrm{PWS}_{U C}$, the problem of deciding whether a subset of alternatives of a partial tournament $G$ is the uncovered set of some completion of $G$, is NP-complete. The proof of this result proceeds by a reduction of SAT and involves the construction of partial tournaments on the basis of formulas in conjunctive normal form. For each propositional variable $p$ and every clause $c$, there is a gadget that is based on the partial tournament $G_{p}$ depicted in Figure $4(i)$.

It is not hard to see that there are exactly two completions of $G_{p}$ with $\left\{p^{-}, p^{+}, 1\right\}$ as the uncovered set. The first, $T^{+}$or positive completion, is depicted in Figure 4(ii) and the other, $T^{-}$or negative completion, in Figure 4(iii). To verify that there are no other ones, consider an arbitrary completion $\left(V, E^{\prime}\right)$ of $G_{p}$. Then, either $\left(\underline{p}^{-}, \underline{p}^{+}\right) \in E^{\prime}$ or $\left(\underline{p}^{+}, \underline{p}^{-}\right) \in E^{\prime}$. In the former case, observe that $\underline{p}^{-}$must be covered by 1 . Hence, $\left(1, p^{-}\right) \in E^{\prime}$ and $\left(\underline{c}, \underline{p}^{-}\right) \in E^{\prime}$. It now follows that $\underline{c}$ is covered by $p^{+}$. Therefore, also $\left(p^{+}, 1\right) \in E^{\prime}$ and $\left(\underline{p}^{+}, \underline{c}\right) \in E^{\prime}$. This entails that $p^{-}$covers $p^{+}$and, with $\left(p^{+}, p^{+}\right) \in E^{\prime}$ we finally obtain $\left(p^{-}, p^{+}\right) \in E^{\prime}$. The resulting tournament is $T^{+}$. By an analogous argument it can be seen that $T^{-}$results if we assume that $\left(\underline{p}^{-}, \underline{p}^{+}\right) \in E^{\prime}$. In the construction below, the positive completion $T^{+}$will correspond to setting propositional variable $p$ to true and the negative completion $T^{-}$to setting $p$ to false.

Besides $\underline{c}$, the construction also involves an alternative $c$ for each clause. How $c$ is related to the other alternatives in $G_{p}$ depends on whether the respective clause contains $p$ or $\bar{p}$ as a literal. As we may assume that no clause contains both $p$ and $\bar{p}$, three cases remain, which 


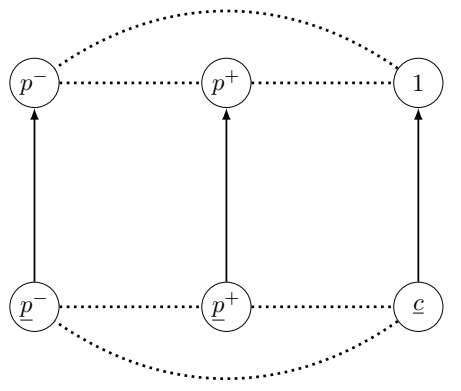

( $i$ ) variable gadget for variable $p$

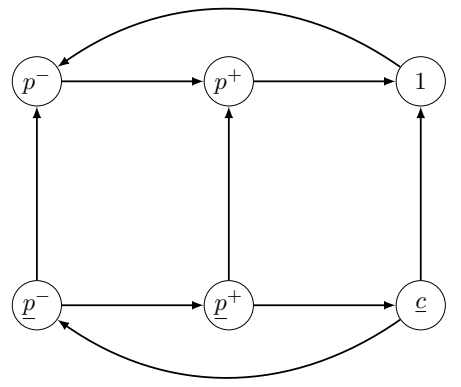

(ii) completion $T^{+}$for $p$ set to true

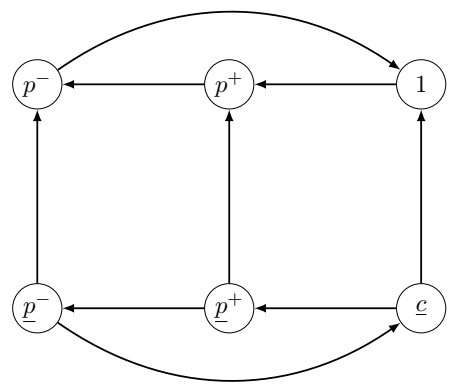

(iii) completion $T^{-}$for $p$ set to false

Figure 4: The partial tournament $G_{p}$ and its only two completions, $T^{+}$and $T^{-}$, for which the uncovered set is given by $\left\{p^{-}, p^{+}, 1\right\}$. Dotted edges are missing and omitted edges point downwards.

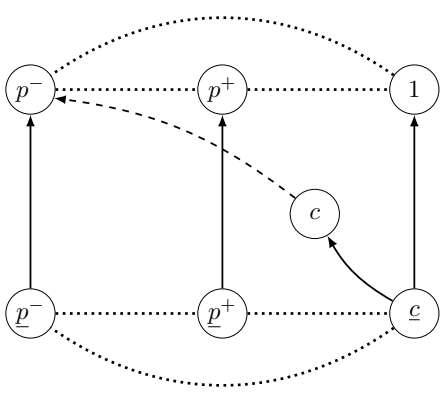

(i) $c$ contains $p$ but not $\bar{p}$

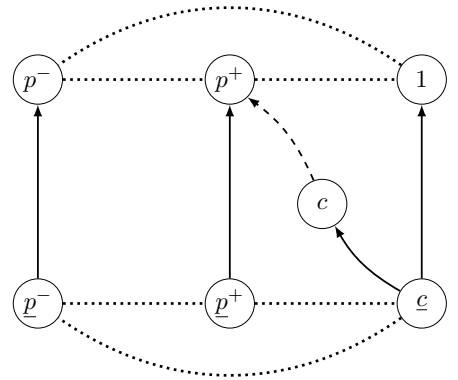

(ii) $c$ contains $\bar{p}$ but not $p$

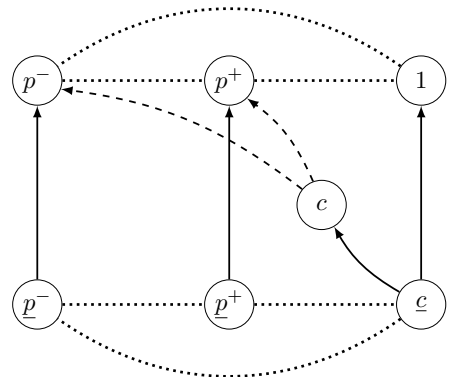

(iii) $c$ contains neither $p$ nor $\bar{p}$

Figure 5: The $G_{p}$-gadget with alternative $c$ added. As in Figure 4, dotted edges are missing and omitted edges point downwards.

are depicted in Figure 5. Some reflection reveals that if a clause contains $p$ as a positive literal, $\underline{c}$ will be covered by $p^{+}$if the partial tournament is completed positively, but not by $p^{-}$if it is completed negatively. Similarly, if the clause contains $\bar{p}$ as a negative literal, $\underline{c}$ will be covered by $p^{-}$if $G_{p}$ is completed negatively, but not by $p^{+}$if $G_{p}$ is completed positively. If $c$ contains neither $p$ nor $\bar{p}$ as a literal, $\underline{c}$ will not be covered by either $p^{+}$or $p^{-}$ irrespective of whether $G_{p}$ is completed positively or negatively.

In the construction below, for every clause, alternative $\underline{c}$ will be covered if and only if the clause contains a literal $p$ and the $G_{p}$-gadget is completed positively or a literal $\bar{q}$ and the $G_{q}$-gadget is completed negatively.

Theorem 7. PWS ${ }_{U C}$ is NP-complete.

Proof. Given a partial tournament $G=(V, E)$, a set $X \subseteq V$, and a completion $T \in[G]$, it can be checked in polynomial time whether $X=U C(T)$. Hence, $\mathrm{PWS}_{U C}$ is obviously in NP.

NP-hardness is shown by a reduction from SAT. Let $\varphi$ be a formula in conjunctive normal form. Without loss of generality we may assume that no clause contains both a 
literal and its negation, that there are at least two clauses, and that every literal occurs in at least one clause. We construct a partial tournament $G_{\varphi}=\left(V_{\varphi}, E_{\varphi}\right)$ as follows. For each propositional variable $p$ we introduce five alternatives denoted $p, p^{-}, p^{+}, p^{-}$, and $p^{+}$. For each clause $c$, we introduce two alternatives denoted $c$ and $\underline{c}$. We also have two auxiliary alternatives denoted by 1 and $\underline{0}$. Thus,

$$
V_{\varphi}=\left\{p, p^{-}, p^{+}, \underline{p}^{-}, \underline{p}^{+}: p \text { is a variable }\right\} \cup\{c, \underline{c}: c \text { is a clause }\} \cup\{1, \underline{0}\} .
$$

We now give a description of the edge set $E_{\varphi}$. For every propositional variable $p$ and every clause $c$ the alternatives $p^{-}, p^{+}, \underline{p}^{-}, \underline{p}^{+}, 1, c$, and $\underline{c}$ are organized as in Figure 5 . The remaining edges are set in such a way as to make the construction work properly. Formally, define the edge set $E_{\varphi}$ such that for every propositional variable $p$ and every clause $c$ :

- $p$ dominates $d$ and $\underline{d}$ for every clause $d$ as well as $q^{-}, q^{+}, \underline{q}^{-}, \underline{q}^{+}$for every $q \neq p$;

- $p^{+}$dominates $\underline{0}, p$, and $\underline{p}^{-}$along with $\underline{q}^{-}, q^{+}$, and $\underline{d}$ for all $q \neq p$ and all clauses $d$. Moreover, for every clause $d$, alternative ${p^{+}}^{-}$dominates alternative $d$ if and only if $p$ occurs as a literal in clause $d$;

- $p^{-}$dominates $\underline{0}, p$, and $\underline{p}^{+}$along with $\underline{q}^{-}, \underline{q}^{+}$, and $\underline{d}$ for all $q \neq p$ and all clauses $d$. Moreover, for every clause $d$, alternative $p^{-}$dominates alternative $d$ if and only if $\bar{p}$ occurs as a literal in clause $d$;

- $\underline{p}^{+}$dominates $\underline{0}, p$, and $p^{+}$;

- $\underline{p}^{-}$dominates $\underline{0}, p$, and $p^{-}$;

- $c$ dominates $\underline{0}, \underline{q}^{-}$, and $\underline{q}^{+}$for every variable $q$, and $\underline{d}$ for every clause $d \neq c$. Moreover, for very variable $q$, alternative $c$ dominates $q^{+}$whenever $c$ does not contain $q$ as a literal, and $q^{-}$if $c$ does not contain $\bar{q}$ as a literal;

- $\underline{c}$ dominates $\underline{0}, c$, and 1 ;

- 1 dominates $\underline{0}$ as well as $q, \underline{q}^{-}$, and $\underline{q}^{+}$for all variables $q$, and $d$ for all clauses $d$;

- $\underline{0}$ dominates alternative $q$ for every variable $q$, otherwise $\underline{0}$ is dominated by all other alternatives.

Moreover, for every variable $p$, the edges among $p^{-}, p^{+}$, and 1 are missing as well as those between $\underline{p}^{-}, \underline{p}^{+}$, and $\underline{d}$ for every clause $d$. Finally, any edges not specified in the above description can be set arbitrarily. For an example of this construction the reader is referred to Figure 6.

Now let

$$
X=\left\{p, p^{-}, p^{+}: p \text { a propositional variable }\right\} \cup\{c: c \text { a clause }\} \cup\{1\} .
$$

Table 2 summarizes which alternatives can reach which other alternatives in at most two steps in $G_{\varphi}$. We thus find that, for every completion $T$ of $G_{\varphi}$, the set $X$ is contained in $U C(T)$ and that $\underline{0}$ is covered by 1 . For propositional variables $p$ and clauses $c$, the alternatives $\underline{p}^{-}, \underline{p}^{+}$, and $\underline{c}$ can only be covered by alternatives from $\left\{p^{-}, p^{+}, 1\right\}$, i.e., whether 


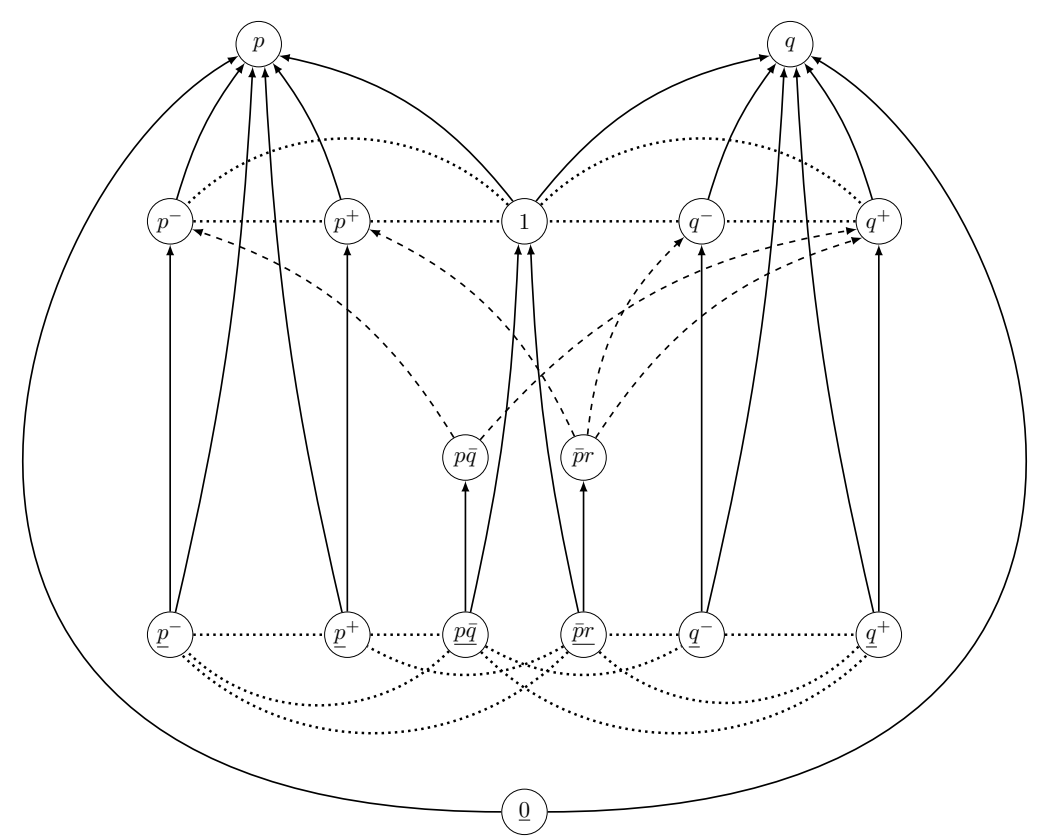

Figure 6: Part of the dominance relation of the partial tournament $G_{\varphi}$ associated with the CNF formula $\varphi=(p \vee \bar{q}) \wedge(\bar{p} \vee r)$. The alternatives $p \bar{q}$ and $\bar{p} r$ represent the two clauses of $\varphi$. The part involving variable $r$, i.e., the alternatives $r, r^{-}, r^{+}, \underline{r}^{-}$, and $\underline{r}^{+}$, has been omitted. The dashed edges are dependent on the clauses of $\varphi$. Omitted edges point downwards or, when on the same level, in an arbitrary direction. Dotted edges are missing.

they are covered only depends on how the subtournament on $\left\{p^{-}, p^{+}, 1, p^{-}, p^{+}, \underline{c}\right\}$ is completed. As we saw in the discussion preceding this theorem, this can be done 'positively' or 'negatively', where a positive completion corresponds to setting variable $p$ to true and a negative completion to setting $p$ to false. We complete the proof by showing that

$$
X=U C(T) \text { for some } T \in\left[G_{\varphi}\right] \text { if and only if } \varphi \text { is satisfiable. }
$$

$(\Leftarrow)$ First assume that $\varphi$ is satisfiable and let $v$ be the satisfying assignment for $\varphi$. For each propositional variable $p$ that $v$ sets to true and each clause $c$, we complete the subtournament on $\left\{p^{-}, p^{+}, 1, \underline{p}^{-}, \underline{p}^{+}, \underline{c}\right\}$ positively, i.e., we add edges $\left(p^{-}, p^{+}\right),\left(p^{+}, 1\right)$, and $\left(1, p^{-}\right)$ as well as $\left(\underline{p}^{-}, p^{+}\right),\left(\underline{p}^{+}, \underline{c}\right)$, and $\left(\underline{c}, p^{-}\right)$. Thus, $p^{-}$is covered by $1, p^{+}$by $p^{-}$, and, provided that $p$ occurs as a literal in $c, \underline{c}$ also by $p^{+}$. Similarly, for each propositional variable $q$ that $v$ sets to false and each clause $c$, we complete the subtournament on $\left\{p^{-}, p^{+}, 1, \underline{p}^{-}, \underline{p}^{+}, \underline{c}\right\}$ negatively, i.e., we add edges $\left(1, q^{+}\right),\left(q^{+}, q^{-}\right)$, and $\left(q^{-}, 1\right)$ as well as $\left(\underline{c}, \underline{q}^{+}\right),\left(\underline{q}^{+}, \underline{q}^{-}\right)$, and $\left(\underline{q}^{-}, \underline{c}\right)$. Accordingly, $\underline{q}^{-}$is covered by $q^{+}, \underline{q}^{+}$by 1 , and, provided that $\bar{q}$ occurs as a literal in $c, \underline{c}$ also by $q^{-}$. Observe that this procedure induces a well-defined completion of $G_{\varphi}$, which we denote by $T_{v}$. As $v$ satisfies $\varphi$, every clause contains a literal $p$ such that $v$ sets $p$ to true or a literal $\bar{q}$ such that $v$ sets $q$ to false. It follows that for every clause $c$, alternative $\underline{c}$ is covered in $T_{v}$. Observe that $\underline{p}^{-}$and $\underline{p}^{+}$are covered in $T_{v}$ irrespective of whether $v$ sets $p$ 


\begin{tabular}{|c|c|c|c|c|c|c|c|c|c|c|c|c|c|c|c|c|}
\hline & $p$ & $q$ & $p^{-}$ & $p^{+}$ & 1 & $q^{-}$ & $q^{+}$ & $c$ & $d$ & $\underline{p}^{-}$ & $\underline{p}^{+}$ & $\underline{c}$ & $\underline{d}$ & $\underline{q}^{-}$ & $\underline{q}^{+}$ & $\underline{0}$ \\
\hline$p$ & . & $q^{-}$ & $c[p]$ & $c[\bar{p}]$ & $\underline{c}$ & . & . & . & . & $c$ & $c$ & . & . & . & . & $c$ \\
\hline$p^{-}$ & . & $\underline{0}$ & . & $\underline{p}^{+}$ & $\underline{c}$ & $p$ & $p$ & $p$ & $p$ & $c[\bar{p}]$ & . & . & . & . & . & . \\
\hline$p^{+}$ & . & $\underline{0}$ & $\underline{p}^{-}$ & & $\underline{c}$ & $p$ & $p$ & $p$ & $p$ & & $c[p]$ & . & . & . & . & . \\
\hline 1 & . & . & $\underline{\bar{p}}^{-}$ & $\underline{p}^{+}$ & . & $\underline{q}^{-}$ & $\underline{q}^{+}$ & . & . & . & . & $p$ & $p$ & . & . & . \\
\hline$c[p]$ & $\underline{0}$ & $\underline{0}$ & 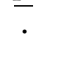 & $\underline{\bar{p}}^{+}$ & $\underline{d}$ & 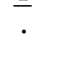 & $\cdot$ & . & $\underline{d}$ & . & . & $p^{-}$ & . & . & . & . \\
\hline$c[\bar{p}]$ & $\underline{0}$ & $\underline{0}$ & $\underline{p}^{-}$ & $=$ & $\underline{d}$ & . & . & . & $\underline{d}$ & & . & $p^{+}$ & . & . & . & . \\
\hline$\underline{p}^{-}$ & . & $\underline{0}$ & . & $\square$ & $\square$ & $p$ & $p$ & $p$ & $p$ & 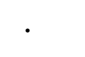 & $p^{-}$ & $p$ & $p$ & $p$ & $p$ & . \\
\hline $\bar{p}^{+}$ & . & $\underline{\overline{0}}$ & $\square$ & . & $\square$ & $p$ & $p$ & $p$ & $p$ & $p^{+}$ & 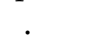 & $p$ & $p$ & $p$ & $p$ & . \\
\hline$\underline{\bar{c}}[p]$ & $\underline{0}$ & $\underline{0}$ & $c$ & $\square$ & . & $c$ & $c$ & 1 & 1 & 1 & 1 & . & $c$ & 1 & 1 & . \\
\hline$\underline{c}[\bar{p}]$ & $\underline{0}$ & $\underline{0}$ & $\square$ & $c$ & . & $c$ & $c$ & 1 & 1 & 1 & 1 & . & $c$ & 1 & 1 & . \\
\hline$\underline{0}$ & & . & $q$ & $q$ & - & $p$ & $p$ & $p$ & $p$ & $q$ & $q$ & $p$ & $p$ & $p$ & $p$ & . \\
\hline
\end{tabular}

Table 2: Table summarizing which types of alternatives reach which other types of alternatives in one or two steps in (all completions of) the partial tournament $G_{\varphi}$. We assume $p$ and $q$ to be distinct variables such that neither $q$ nor $\bar{q}$ occurs as a literal in $c$. Furthermore, $c$ and $d$ are assumed to be distinct clauses, where $c[p]$ denotes clause $c$ on the understanding that $p$ occurs as a literal in $c$. Similarly, $c[\bar{p}]$ denotes clause $c$ on the understanding that $\bar{p}$ occurs as a literal in $c$. An alternative $x$ in the entry for row $r$ and column $c$ means that $r$ can reach $c$ via $x$. If the entry is a dot ("."), $r$ can reach $c$ directly, i.e., in one or zero steps. A box (" $\square$ ") signifies that it depends on how $G_{\varphi}$ is being completed whether and via which alternative $r$ can reach $c$. The minus ("-") in the entry for $\underline{0}$ and 1 means that $\underline{0}$ cannot reach 1 in at most two steps, no matter how $G_{\varphi}$ is completed. Thus, $\underline{0}$ is covered by 1 in every completion of $G_{\varphi}$. We may assume that no clause contains both a literal and its negation, that there are at least two clauses, and that every literal occurs in at least one clause.

to true or false. Hence, $\underline{c}, \underline{p}^{+}, \underline{p}^{-} \notin U C\left(T_{v}\right)$. Recalling that 1 covers $\underline{0}$ and $X \subseteq U C(T)$ for all completions $T$ of $G_{\varphi}$, we may conclude that $U C\left(T_{v}\right)=X$, as desired.

$(\Rightarrow)$ For the opposite direction, assume that there is some completion $T$ of $G_{\varphi}$ such that for every propositional variable $p$ and for every clause $c$, alternatives $p^{-}, p^{+}$, and $\underline{c}$ are covered in $T$, i.e., such that $U C(T)=X$. Define assignment $v_{T}$ such that it sets propositional variable $p$ to true if there is some clause $c$ containing $p$ as a literal such that $p^{+}$covers $\underline{c}$ in $T$ and sets $p$ to false, otherwise. Observe that $v_{T}$ is a well-defined assignment.

We now show that $v_{T}$ satisfies every clause in $\varphi$ and hence $\varphi$ itself as well. To this end consider an arbitrary clause $c$. By assumption, $\underline{c}$ is covered by some alternative $x$. Recall that $\underline{c}$ reaches all alternatives in at most two steps except alternatives $p^{+}$such that $p$ occurs as a literal in $c$ and alternatives $q^{-}$such that $\bar{q}$ occurs as a literal in $c$ (also see Table 2). Hence, either $x=p^{+}$for some variable $p$ occurring as a literal in $c$ or $x=q^{-}$for some variable $q$ such that $\bar{q}$ occurs as a literal in $c$.

If the former, $v_{T}$ sets $p$ to true and consequently also satisfies clause $c$. If the latter, we have to demonstrate that $v_{T}$ sets $q$ to false and in that way satisfies clause $c$. It suffices to 


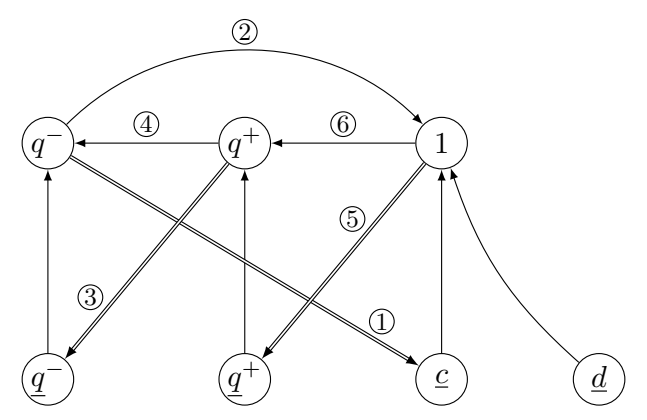

Figure 7: Illustration of the concluding argument of the proof of Theorem 7. A double edge from alternative $x$ to alternative $y$ indicates that $x$ covers $y$. The numbers some of the edges are labelled with correspond to the order in which their existence is demonstrated in the proof of Theorem 7 .

show that there is no clause $d$ such that $q^{+}$covers $\underline{d}$ in $T$. To this end, consider an arbitrary clause $d$. We prove that $q^{+}$does not cover $\underline{d}$ and refer to Figure 7 for an illustration of our reasoning. Let $E_{T}$ denote the edge set of $\bar{T}$. As $T$ extends $G_{\varphi}$, obviously $E_{\varphi} \subseteq E_{T}$. First recall that we have assumed that $q^{-}$covers $\underline{c}$ in $T$. As $(\underline{c}, 1) \in E_{\varphi}$, then also $\left(q^{-}, 1\right) \in E_{T}$. Since $\left(q^{-}, q^{-}\right) \in E_{\varphi}$, it can therefore not be the case that 1 covers $q^{-}$in $T$. Reaching every other alternative in at most two steps in $G_{\varphi}$, alternative $q^{-}$must therefore be covered by $q^{+}$ in $T$. As $\left(q^{-}, q^{-}\right) \in E_{\varphi}$, it now follows that $\left(q^{+}, q^{-}\right) \in \overline{E_{T}}$. Since, moreover, $\left(q^{+}, q^{+}\right) \in E_{\varphi}$, we also have that $q^{-}$does not cover $q^{+}$in $T$. Rather, $\underline{q}^{+}$reaches every alternative except 1 in at most two steps in $T$. It follows that $\underline{q}^{+}$is covered by 1 . Moreover, since $\left(\underline{q}^{+}, q^{+}\right) \in E_{\varphi}$, also $\left(1, q^{+}\right) \in E_{T}$. Now consider alternative $\underline{d}$ and observe that, by construction, $(\underline{d}, 1) \in E_{\varphi}$. Thus, $\underline{d}$ can reach $q^{+}$in two steps and we may conclude that $q^{+}$does not cover $\underline{d}$ in $T$. It follows that $v_{T}$ sets $q$ to false and thus satisfies $c$, as desired.

\section{Weighted Tournament Solutions}

We now turn to weighted tournaments, and in particular consider the solution concepts Borda, maximin, and ranked pairs.

\subsection{Borda}

The Borda solution $(B O)$ is typically used in a voting context with a set $N$ of $n$ voters, where each voter $i$ is equipped with a linear ranking $\succ_{i}$ as its individual preference. Then, each alternative receives $|V|-1$ points for each time it is ranked first by a voter, $|V|-2$ points for each time it is ranked second, and so forth. The total number of points of an alternative $x$ constitute its Borda score $s_{B O}\left(x,\left(\succ_{i}\right)_{i \in N}\right)$ which can be written as

$$
s_{B O}\left(x,\left(\succ_{i}\right)_{i \in N}\right)=\sum_{i \in N}\left|\left\{y \in V: x \succ_{i} y\right\}\right|
$$


More generally, the Borda solution can be extended to $n$-weighted tournaments where the Borda scores are defined as

$$
s_{B O}(x,(V, w))=\sum_{y \in V \backslash\{x\}} w(x, y)
$$

and $B O(V, w)$ again chooses those alternatives with maximum Borda score. This subsumes the voting setting because

$$
s_{B O}\left(x,\left(\succ_{i}\right)_{i \in N}\right)=\sum_{y \in V}\left|\left\{i \in N: x \succ_{i} y\right\}\right|=s_{B O}(x,(V, w))
$$

if the weight on the edge from $x$ to $y$ is defined to be the number of voters who rank $x$ higher than $y$, i.e.,

$$
w(x, y)=\left|\left\{i \in N: x \succ_{i} y\right\}\right| .
$$

Before we proceed, we define the notion of a $b$-matching, which will be used in the proofs of several of our results in this section. Let $H=\left(V_{H}, E_{H}\right)$ be an undirected graph with vertex capacities $b: V_{H} \rightarrow \mathbb{N}_{0}$. Then, a $b$-matching of $H$ is a function $m: E_{H} \rightarrow \mathbb{N}_{0}$ such that for all $v \in V_{H}$,

$$
\sum_{e \in\left\{e^{\prime} \in E_{H}: v \in e^{\prime}\right\}} m(e) \leq b(v) .
$$

The size of $b$-matching $m$ is defined as $\sum_{e \in E_{H}} m(e)$. It is easy to see that if $b(v)=1$ for all $v \in V_{H}$, then a maximum-size $b$-matching is equivalent to a maximum-cardinality matching. In a $b$-matching problem with upper and lower bounds, there further is a function $a: V_{H} \rightarrow \mathbb{N}_{0}$. A feasible $b$-matching then is a function $m: E_{H} \rightarrow \mathbb{N}_{0}$ such that

$$
a(v) \leq \sum_{e \in\left\{e^{\prime} \in E_{H}: v \in e^{\prime}\right\}} m(e) \leq b(v)
$$

If $H$ is bipartite, then the problem of computing a maximum-size feasible $b$-matching with lower and upper bounds can be solved in strongly polynomial time (Schrijver, 2003, ch. 21). We will use this result to show that $\mathrm{PW}_{B O}$ and $\mathrm{PWS}_{B O}$ can both be solved in polynomial time. While the following result for $\mathrm{PW}_{B O}$ can also be shown using Theorem 6.1 of Kern and Paulusma (2004), we still give a direct proof that will then be extended to $\mathrm{PWS}_{B O}$.

Theorem 8. $\mathrm{PW}_{B O}$ can be solved in polynomial time.

Proof. Observe that $B O$ satisfies the following (weak) monotonicity property: making a winner $x$ stronger by increasing weight on an edge to another alternative, cannot make $x$ a losing alternative.

Let $G=(V, w)$ be a partial $n$-weighted tournament, $x \in V$. By the previous observation, $x \in P W_{B O}(G)$ if and only if $x \in P W_{B O}\left(G^{x \rightarrow}\right)$. Therefore, we can assume w.l.o.g that $G=G^{x \rightarrow}$, i.e., all edges incident to $x$ are completely specified already. Moreover, if there exists a $y \in V \backslash\{x\}$ such that $s_{B O}\left(y, G^{x \rightarrow}\right)>s_{B O}\left(x, G^{x \rightarrow}\right)$, then we already know that $x \notin P W_{B O}(G)$. We thus assume that $s_{B O}\left(y, G^{x \rightarrow}\right) \leq s_{B O}\left(x, G^{x \rightarrow}\right)$ for all $y \in V \backslash\{x\}$. 
We give a polynomial-time algorithm for checking whether $x \in P W_{B O}\left(G^{x \rightarrow}\right)$ via a reduction to the problem of computing a maximum-size $b$-matching of a bipartite graph.

Let $s^{*}=s_{B O}\left(x, G^{x \rightarrow}\right)$ be the Borda score of $x$ in $G^{x \rightarrow}$. We construct a bipartite graph $H=\left(V_{H}, E_{H}\right)$ with vertices

$$
\begin{aligned}
& V_{H}=V \backslash\{x\} \cup E^{x}, \text { where } \\
& E^{x}=\{\{i, j\} \subseteq V \backslash\{x\}: i \neq j\}
\end{aligned}
$$

and edges

$$
E_{H}=\{\{i,\{i, j\}\}:\{i, j\} \subseteq V \backslash\{x\}, i \neq j\} .
$$

We further define vertex capacities $b: V_{H} \rightarrow \mathbb{N}_{0}$ such that

$$
\begin{aligned}
b(\{i, j\}) & =n-w(i, j)-w(j, i) \text { for }\{i, j\} \in E^{x} \text { and } \\
b(v) & =s^{*}-s_{B O}\left(v, G^{x \rightarrow}\right) \text { for } v \in V \backslash\{x\} .
\end{aligned}
$$

Now observe that in any completion $T=\left(V, w^{\prime}\right) \in\left[G^{x \rightarrow}\right], w^{\prime}(i, j)+w^{\prime}(j, i)=n$ for all $i, j \in V$ with $i \neq j$. The sum of the Borda scores in $T$ is therefore $n|V|(|V|-1) / 2$. Some of the weight has already been used up in $G^{x \rightarrow}$; the weight which has not yet been used up is equal to

$$
\alpha=n|V|(|V|-1) / 2-\sum_{v \in V} s_{B O}\left(v, G^{x \rightarrow}\right) .
$$

We claim that $x \in P W_{B O}\left(G^{x \rightarrow}\right)$ if and only if $H$ has a $b$-matching of size at least $\alpha$.

$(\Rightarrow)$ Let $T=\left(V, w^{\prime}\right) \in\left[G^{x \rightarrow}\right]$ be a completion with $x \in B O(T)$. Consider the $b$ matching $m$ with $m(i,\{i, j\})=w^{\prime}(i, j)-w(i, j)$. We verify that $m$ is a feasible $b$-matching. Let $v \in V_{H}$. If $v \in V \backslash\{x\}$, we have that

$$
\sum_{e \in\left\{e^{\prime} \in E_{H}: v \in e^{\prime}\right\}} m(e)=s_{B O}(v, T)-s_{B O}\left(v, G^{x \rightarrow}\right) \leq s^{*}-s_{B O}\left(v, G^{x \rightarrow}\right)=b(v) .
$$

Otherwise, $v=\{i, j\} \in E^{x}$ and

$$
\sum_{e \in\left\{e^{\prime} \in E_{H}:\{i, j\} \in e^{\prime}\right\}} m(e)=m(\{i,\{i, j\}\})+m(\{j,\{i, j\}\})=n-w(i, j)-w(j, i)=b(\{i, j\}) .
$$

As the size of $m$ is

$$
\sum_{e \in E_{H}} m(e)=\sum_{i \neq j}\left(w^{\prime}(i, j)+w^{\prime}(j, i)-w(i, j)-w(j, i)\right)=\sum_{i \neq j} n-\sum_{i \in V} \sum_{j \in V \backslash\{i\}} w(i, j)=\alpha
$$

the statement is shown.

$(\Leftarrow)$ For the other direction, assume that a feasible $b$-matching of size at least $\alpha$ exists. We construct a completion $T=\left(V, w^{\prime}\right) \in\left[G^{x \rightarrow}\right]$ with $x \in B O(T)$. Let

$$
\begin{aligned}
& w^{\prime}(i, j)=m(i,\{i, j\})+w(i, j) \\
& w^{\prime}(x, i)=w(x, i) \text {, and } w^{\prime}(i, x)=w(i, x) \quad \text { for } i \in V \backslash\{x\} \text {. }
\end{aligned}
$$


As $w(i, j) \leq w^{\prime}(i, j)$ and $w^{\prime}(i, j)+w^{\prime}(j, i) \leq w(i, j)+w(j, i)+b(\{i, j\})=n$ for all $\{i, j\} \subseteq V$, $T$ is an extension of $G^{x \rightarrow}$. From

$$
\alpha=\sum_{\{i, j\} E^{x}} b(\{i, j\}) \geq \sum_{e \in E_{H}} m(e)=\sum_{i \neq j} \geq \alpha,
$$

we know that the upper capacities $b(\{i, j\})$ of all $\{i, j\} \in E^{x}$ are exactly met by $m$ (and that there cannot be a matching with size more than $\alpha$ ). This implies that

$$
w^{\prime}(i, j)+w^{\prime}(j, i)=w(i, j)+w(j, i)+b(\{i, j\})=n,
$$

showing that $T$ is indeed a completion of $G^{x \rightarrow}$.

Since $H$ can be constructed efficiently, and since a maximum-size $b$-matching can be computed in strongly polynomial time, our algorithm runs in polynomial time.

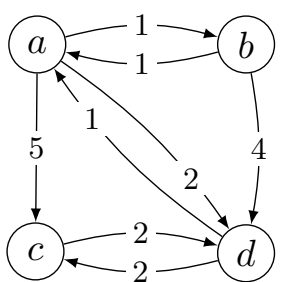

(i) A partial 5-weighted tournament $G$.

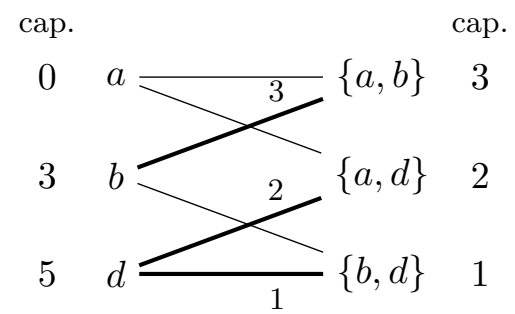

(iii) The constructed bipartite graph $H$ for target Borda score $s^{*}=s_{B O}\left(c, G^{c \rightarrow}\right)=8$. Capacities are given next to the vertices. Thick edges with weights indicate the unique maximum $b$-matching.

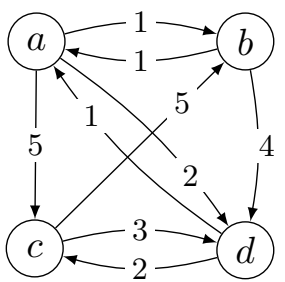

(ii) The partial tournament $G^{c \rightarrow}$.

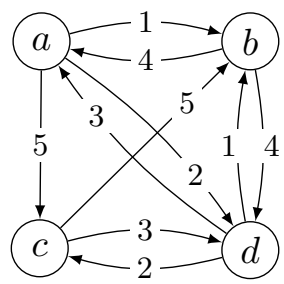

(iv) The completion $T$ of $G$ that corresponds to the maximum $b$-matching. In this case, $B O(T)=\{a, b, c\}$.

Figure 8: Illustration of the algorithm for checking whether an alternative $c$ is contained in $P W_{B O}(G)$ for a partial 5-weighted tournament $G$.

Figure 8 illustrates the described steps for determining whether an alternative is contained in $P W_{B O}(G)$.

This idea can be extended to a polynomial-time algorithm for $\mathrm{PWS}_{B O}$ where we use a similar construction for a given $G=(V, w)$, a candidate set $X \subseteq V$ and a target Borda score $s^{*}$. Binary search can be used to efficiently search the interval of possible target scores. 
Theorem 9. $\mathrm{PWS}_{B O}$ can be solved in polynomial time.

Proof. Let $G=(V, w)$ be a partial $n$-weighted tournament, and $X \subseteq V$. We give a polynomial-time algorithm for checking whether $X \in P W S_{B O}(G)$, via a bisection method and a reduction to the problem of computing a maximum $b$-matching of a graph with lower and upper bounds.

Assume that there is a target Borda score $s^{*}$ and a completion $T \in[G]$ with $X \in$ $P W S_{B O}(T)$ and $s_{B O}(x, T)=s^{*}$ for all $x \in X$. Then, the maximum possible Borda score of an alternative not in $X$ is $s^{*}-1$.

For a given target Borda score $s^{*}$, we construct a bipartite graph $H=\left(V_{H}, E_{H}\right)$ with vertices $V_{H}=V \cup E^{x}$, where

$$
E^{x}=\{\{i, j\} \subseteq V: i \neq j\}
$$

and edges

$$
E_{H}=\{\{i,\{i, j\}\}:\{i, j\} \subseteq V, i \neq j, w(i, j)+w(j, i)<n\} .
$$

Only the lower bounds $a_{s^{*}}: V_{H} \rightarrow \mathbb{N}_{0}$ and upper bounds $b_{s^{*}}: V_{H} \rightarrow \mathbb{N}_{0}$ depend on $s^{*}$ and are defined as follows. For vertices $x \in X$, lower and upper bounds coincide and are given by

$$
a_{s^{*}}(x)=b_{s^{*}}(x)=s^{*}-s_{B O}(x, G) .
$$

All other vertices $v \in V_{H} \backslash X$ have a lower bound of $a_{s^{*}}(v)=0$. Upper bounds for these vertices are defined such that

$$
b_{s^{*}}(v)=s^{*}-s_{B O}(v, G)-1
$$

for $v \in V \backslash X$, and

$$
b_{s^{*}}(\{i, j\})=n-w(i, j)-w(j, i)
$$

for $\{i, j\} \in E^{x}$.

As in the proof of Theorem 8, it holds that a feasible $b$-matching in $H$ corresponds to an extension of $G$. Such an extension is a completion $T \in[G]$ if and only if the $b$-matching has size

$$
\alpha=n|V|(|V|-1) / 2-\sum_{v \in V} s_{B O}(v, G),
$$

which equals the weight not yet used up in $G$. Then, $T$ satisfies $X \in P W S_{B O}(T)$ and $s_{B O}(x, T)=s^{*}$ for all $x \in X$. If, on the other hand, no $s^{*}$ gives rise to a graph that has a $b$-matching of size $\alpha$, then $X \notin P W S_{B O}(G)$.

In order to obtain a polynomial-time algorithm, we need to check whether there exists a target score $s^{*}$ for which the corresponding graph $H$ with upper and lower bounds admits a $b$-matching of size $\alpha$. It is easily verified that any such $s^{*}$ is contained in the integer interval

$$
I=\left[\max _{x \in X} s_{B O}(x, G), n(|V|-1)\right]
$$

Observe that $|I|$ depends on $n$ and thus is not polynomially bounded in the size of $G$. Checking every integer $s \in I$ is therefore not feasible in polynomial time. However, we now show that we can perform binary search in order to find $s^{*}$ efficiently. We need the following 
two observations about the interval $I$. For $s \in I$, we say that $s$ admits a feasible b-matching if the corresponding graph $H$ has a feasible $b$-matching.

First, if an $s^{\prime} \in I$ admits a feasible $b$-matching, then every $s^{\prime \prime} \in I$ with $s^{\prime \prime} \leq s^{\prime}$ also admits a feasible $b$-matching. This is because removing all weight from edges that exceeds the (reduced) upper bounds gives a feasible $b$-matching for $s^{\prime \prime}$.

Second, with $s^{\prime}$ as before and $\alpha^{\prime}$ the size of the corresponding maximum feasible $b$ matching $m^{\prime}$, there cannot be an $s^{\prime \prime} \in I$ with $s^{\prime \prime} \geq s^{\prime}$ such that the size $\alpha^{\prime \prime}$ of a maximum feasible $b$-matching $m^{\prime \prime}$ for $s^{\prime \prime}$ is smaller than $\alpha^{\prime}$. This is because either $(i)$ no such $m^{\prime \prime}$ exists since not all lower bounds can be met, or (ii) such an $m^{\prime \prime}$ exists and its size is at least $\alpha^{\prime}$. To see the latter, note that a decrease in the size of a maximum feasible matching cannot be caused by upper bounds as $b_{s^{\prime \prime}}(v) \geq b_{s^{\prime}}(v)$ for all $v \in V_{H}$. It remains to be shown that the increase in $a_{s^{\prime \prime}}(v)$ for $v \in X$ does not result in a smaller maximum $b$-matching. Since the weight of all edges incident to a vertex in $X$ in the $b$-matching is completely determined by the bounds and increases from $m^{\prime}$ to $m^{\prime \prime}$, a total decrease in size can only be due to edges $\{j,\{i, j\}\}$ with $i \in V \backslash X, j \in V$ whose weight is bounded by $b_{s^{\prime \prime}}(\{i, j\})-m^{\prime \prime}(i,\{i, j\})$. But then,

$$
m^{\prime \prime}(i,\{i, j\})+m^{\prime \prime}(j,\{i, j\})=b_{s^{\prime \prime}}(\{i, j\}) \geq b_{s^{\prime}}(\{i, j\}) \geq m^{\prime}(i,\{i, j\})+m^{\prime}(j,\{i, j\})
$$

and therefore $\alpha^{\prime \prime} \geq \alpha^{\prime}$.

These two observations show that $I$ can be partitioned into two non-overlapping integer intervals $I_{1}$ and $I_{2}$. Here, each $s \in I_{1}$ admits a feasible $b$-matching whose size increases when $s$ grows, whereas each $s \in I_{2}$ does not admit a feasible $b$-matchings. Therefore, either $I_{1}$ is empty and the desired $s^{*}$ does not exist, or $s^{*}=\max \left(I_{1}\right)$.

We can check the existence of $s^{*}$ with the following binary search algorithm. Let $\left[I_{\min }, I_{\max }\right]$ be an interval that is initialized to $I=\left[\max _{x \in X} s_{B O}(x, G), n(|V|-1)\right]$. Consider the median value $s$ of this interval. If the corresponding graph $H$ has no feasible $b$-matching, continue with the interval $\left[I_{\min }, s-1\right]$. Otherwise, if the maximum feasible $b$-matching has size at least $\alpha$, return "yes". If its size is less than $\alpha$, continue with $\left[s+1, I_{\max }\right]$. If $\left[I_{\min }, I_{\max }\right]$ is empty, return "no."

The number of queries of this algorithm is bounded by $\left\lceil\log _{2}|I|\right\rceil \leq\left\lceil\log _{2} n|V|\right\rceil$ and, therefore, polynomial in the size of $G$.

To conclude this section, we show that $\mathrm{NW}_{B O}$ can be solved in polynomial time as well. It is worth noting that this result does not follow directly from the polynomial-time result for $\mathrm{NW}_{B O}$ for the case of preference profiles (Xia \& Conitzer, 2011).

Theorem 10. $\mathrm{NW}_{B O}$ can be solved in polynomial time.

Proof. Let $G=(V, w)$ be a partial weighted tournament, $x \in V$. We give a polynomial-time algorithm for checking whether $x \in N W_{B O}(G)$.

Let $\hat{G}=G^{x \leftarrow}$. We want to check whether some other alternative $y \in V \backslash\{x\}$ can achieve a Borda score of more than $s^{*}=s_{B O}(x, \hat{G})$. This can be done separately for each $y \in V \backslash\{x\}$ by reinforcing it as much as possible in $\hat{G}$. If for some $y, s_{B O}\left(y, \hat{G}^{y \rightarrow}\right)>s^{*}$, then $x \notin N W_{B O}(G)$. If, on the other hand, $s_{B O}\left(y, \hat{G}^{y \rightarrow}\right) \leq s^{*}$ for all $y \in V \backslash\{x\}$, then $x \in N W_{B O}(G)$. 
As an example, consider the partial 5-weighted tournament $G$ in Figure $8(i)$. The fact that $\{a, b, c\} \subseteq P W_{B O}(G)$ follows already from the completion shown in Figure 8(iv). Also note that this was the only completion in which $c$ was chosen. Alternative $d$ is not a possible Borda winner since $s_{B O}\left(d, G^{d \rightarrow}\right)=7<8=s_{B O}(a, G)$. To determine $P W S_{B O}(G)$, we still have to check which subsets of $\{a, b, c\}$ are possible winning sets. For singletons, it is easy to see that only $\{a\}$ and $\{b\}$ are in $P W S_{B O}(G)$. For $\{a, b\}$, we could employ the binary search method described in Theorem 9 . Here, we just argue that moving one unit of weight from $(c, d)$ to $(d, c)$ in the completion shown in Figure $8(i v)$ gives a completion in which $\{a, b\}$ is the winning set. For $N W_{B O}(G)$, it is straightforward to check that no alternative is a necessary Borda winner. Altogether, we have that

$$
\begin{aligned}
P W_{B O}(G) & =\{a, b, c\} \\
N W_{B O}(G) & =\emptyset, \text { and } \\
P W S_{B O}(G) & =\{\{a\},\{b\},\{a, b\},\{a, b, c\}\} .
\end{aligned}
$$

\subsection{Maximin}

The maximin score $s_{M M}(x, T)$ of an alternative $x$ in a weighted tournament $T=(V, w)$, is given by its worst pairwise comparison, i.e., $s_{M M}(x, T)=\min _{y \in V \backslash\{x\}} w(x, y)$. The maximin solution, also known as Simpson's method and denoted by $M M$, returns the set of all alternatives with the highest maximin score.

As an example, consider the partial 5-weighted tournament depicted in Figure 9( $i)$. It is easy to see that $a$ (or $b$ ) are the unique maximin winners in all completions of $G^{a \rightarrow}$ (or $G^{b \rightarrow}$ ). Also, $c$ cannot be a possible maximin winner as it will always have a maximin score of 0 whereas $a$ always has at least 1 . Similarly, alternative $d$ can never have a higher maximin score than $a$. Figure 9 (iii) shows a completion in which $\{a, d\}$ is the set of maximin winners. If one unit of weight is shifted from $(c, b)$ to $(b, c)$, the resulting completion has $\{a, b, d\}$ as the maximin winners. It is also straightforward to find a completion of $G^{\{a, b\}} \rightarrow$ with $\{a, b\}$ as the set of maximin winners. It is easy to verify that no alternative is a necessary maximin winner.

Together, this gives

$$
\begin{aligned}
P W_{M M}(G) & =\{a, b, d\}, \\
N W_{M M}(G) & =\emptyset, \text { and } \\
P W S_{M M}(G) & =\{\{a\},\{b\},\{a, b\},\{a, d\},\{a, b, d\}\} .
\end{aligned}
$$

We first show that $\mathrm{PW}_{M M}$ is polynomial-time solvable by reducing it to the problem of finding a maximum-cardinality matching of a graph.

Theorem 11. $\mathrm{PW}_{M M}$ can be solved in polynomial time.

Proof. We show how to check whether $x \in P W_{M M}(G)$ for a partial $n$-weighted tournament $G=(V, w)$. Consider the graph $G^{x \rightarrow}=\left(V, w^{x \rightarrow}\right)$. Then, $s_{M M}\left(x, G^{x \rightarrow}\right)$ is the best possible maximin score $x$ can get among all completions of $G$. If $s_{M M}\left(x, G^{x \rightarrow}\right) \geq \frac{n}{2}$, then we have $s_{M M}(y, T) \leq w^{x \rightarrow}(y, x) \leq \frac{n}{2}$ for every $y \in V \backslash\{x\}$ and every completion $T \in\left[G^{x \rightarrow}\right]$, and therefore $x \in P W_{M M}(G)$. 


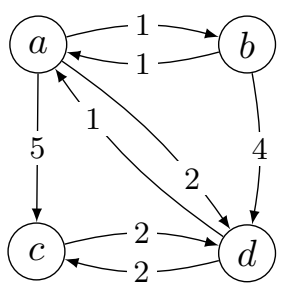

(i) A partial 5-weighted tournament $G$.

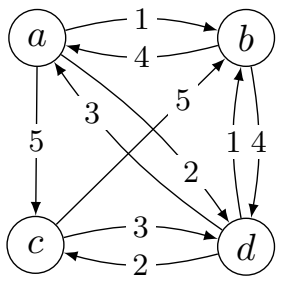

(iii) A completion $T$ of $G$ that could be obtained from the matching. Indeed, $M M(T)=\{a, d\}$ with $s_{M M}(T)=1$.

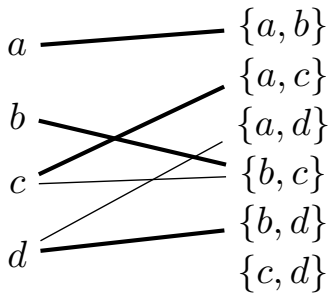

(ii) The constructed bipartite graph $H^{s^{*}}$ for $s^{*}=1$ and $X=\{a, d\}$ as in the proof of Theorem 12. A maximum-cardinality matching is given by the thick edges.

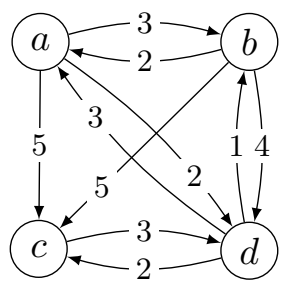

(iv) This completion $T$ of $G$ is a witness for $\{a, b\} \in P W S_{M M}(G)$.

Figure 9: Example of a 5-weighted partial tournament and completions relevant for possible maximin winners.

Now consider $s_{M M}\left(x, G^{x \rightarrow}\right)<\frac{n}{2}$. We will reduce the problem of checking whether $x \in$ $P W_{M M}(G)$ to that of finding a maximum-cardinality matching of an undirected unweighted graph, which is known to be solvable in polynomial time (Edmonds, 1965). We want to find a completion $T \in\left[G^{x \rightarrow}\right]$ such that $s_{M M}(x, T) \geq s_{M M}(y, T)$ for all $y \in V \backslash\{x\}$. In other words, we want to complete the weights on edges between alternatives in $V \backslash\{x\}$ in such a balanced way so that $x$ is still a winner. If there exists a $y \in V \backslash\{x\}$ such that $s_{M M}\left(y, G^{x \rightarrow}\right)>s_{M M}\left(x, G^{x \rightarrow}\right)$, then we already know that $x \notin P W_{M M}(G)$. Otherwise, each $y \in V \backslash\{x\}$ derives its maximin score from at least one particular edge $(y, z)$ where $z \in V \backslash\{x, y\}$ and $w(y, z) \leq s_{M M}\left(x, G^{x \rightarrow}\right)$. Moreover, it is clear that in any completion, $y$ and $z$ cannot both achieve a maximin score of less than $s_{M M}\left(x, G^{x \rightarrow}\right)$ from edges $(y, z)$ and $(z, y)$ at the same time. Let $H=\left(V_{H}, E_{H}\right)$ be an undirected and unweighted graph with vertices

$$
V_{H}=V \backslash\{x\} \cup\{\{i, j\} \subseteq V: i \neq j\}
$$

and edges

$$
E_{H}=\left\{\{i,\{i, j\}\}: i \in V \backslash\{x\}, j \in V \backslash\{i\}, w^{x \rightarrow}(i, j) \leq s_{M M}\left(x, G^{x \rightarrow}\right)\right\} .
$$

In this way, if $i$ is matched to $\{i, j\}$ in $H$, then $i$ derives a maximin score of less than or equal to $s_{M M}\left(x, G^{x \rightarrow}\right)$ from his comparison with $j$. Clearly, the size of $H$ is polynomial 
in the size of $G$. We show that $x \in P W_{M M}(G)$ if and only if there exists a matching of cardinality $|V|-1$ in $H$.

$(\Rightarrow)$ First, assume that $x \in P W_{M M}(G)$. Then there exists a completion $T=\left(V, w^{\prime}\right)$ of $G^{x \rightarrow}$ in which the maximin score of each $y \in V \backslash\{x\}$ is at most $s_{M M}\left(x, G^{x \rightarrow}\right)<\frac{n}{2}$. If alternative $i$ derives its maximin score from a comparison with $j \neq i \in V \backslash\{x\}$, i.e., $s_{M M}(i, T)=w^{\prime}(i, j)$, then $j$ cannot derive its maximin score from a comparison with $i$ because $w^{\prime}(j, i) \geq n-s_{M M}\left(x, G^{x \rightarrow}\right)$ implies $w^{\prime}(j, i)>\frac{n}{2}$. Therefore, in $H$, each $i \in V_{H} \cap V$ can be matched to a vertex $\{i, j\} \in V_{H}$ such that $\{i, j\}$ is not matched to any other vertex in $V_{H}$. The resulting matching in $H$ has cardinality $|V|-1$.

$(\Leftarrow)$ Now, assume that there exists a matching $M$ of cardinality $|V|-1$ in $H$. Then, each $i \in V \backslash\{x\}$ has to be matched to an $\{i, j\}$ where $w(i, j) \leq s_{M M}\left(x, G^{x \rightarrow}\right)$. Consider a completion $T=\left(V, w^{\prime}\right) \in\left[G^{x \rightarrow}\right]$ in which for all $(i, j) \in V \times V$ such that $\{i,\{i, j\}\} \in M$, we set $w^{\prime}(i, j)=w(i, j)$ and $w^{\prime}(j, i)=n-w(i, j)$. Moreover, the weights of all other edges in $T$ are set by any arbitrary completion of edges in $G^{x \rightarrow}$. Clearly, $T$ is a proper completion of $G^{x \rightarrow}$ and therefore of $G$. In $T$, the maximin score of each $y \in V \backslash\{x\}$ is less than or equal to the maximin score of $x$. Therefore $x \in M M(T)$ which implies that $x \in P W_{M M}(G)$.

Next, we show that $P W S_{M M}$ can be solved in polynomial time. The proof proceeds by identifying the maximin values that could potentially be achieved simultaneously by all elements of the set in question, and solving the problem for each of these values using similar techniques as in the proof of Theorem 11. Only a polynomially bounded number of problems need to be considered.

Theorem 12. $P W S_{M M}$ can be solved in polynomial time.

Proof. Let $G=(V, w)$ be a partial $n$-weighted tournament and $X \subseteq V$. We give a polynomial-time algorithm for checking whether $X \in P W S_{M M}(G)$.

If $X \in P W S_{M M}(G)$ there must be a completion $T \in[G]$ and $s^{*} \in\{0, \ldots, n\}$ such that $s_{M M}(x, T)=s^{*}$ for all $x \in X$ and $s_{M M}(i, T)<s^{*}$ for all $y \in V \backslash X$.

First, we note that if $s^{*}>n-w(j, i)$ for some $i \in X, j \in V$ or $s^{*} \leq w(i, j)$ for some $i \notin X, j \in V$, then $X \notin P W S_{M M}(G)$. Therefore, we assume that

$$
\begin{gathered}
n-w(j, i) \geq s^{*} \text { for all } i \in X, j \in V \text { and } \\
w(i, j)<s^{*} \text { for all } i \notin X, j \in V .
\end{gathered}
$$

We treat the cases $s^{*}>\frac{n}{2}, s^{*}=\frac{n}{2}$, and $s^{*}<\frac{n}{2}$ separately.

Case 1: $s^{*}>\frac{n}{2}$. Then, $X \in P W S_{M M}$ only if $X$ is a singleton, and for each $x \in V$, whether $\{x\} \in P W S_{M M}$ with maximin score $s^{*}$ can be checked easily.

Case 2: $\quad s^{*}=\frac{n}{2}$. With the assumptions above, we can define $G^{\prime}=\left(V, w^{\prime}\right)$ as an extension of $G^{X \rightarrow}$ with $w^{\prime}(i, j)=w^{\prime}(j, i)=\frac{n}{2}=s^{*}$ for all $i, j \in X$. Note that in every completion $T$ of $G^{\prime}, s_{M M}(i, T)=s^{*}$ for all $i \in X$ and that $X \in P W S_{M M}(G)$ with maximin score $\frac{n}{2}$ in the corresponding completion if and only if $X \in P W S_{M M}\left(G^{\prime}\right)$ with the same maximin score in the respective completion. 
In addition, we need to check whether alternatives not in $X$ can be forced to have a strictly smaller maximin score than $\frac{n}{2}$. To this end, construct an unweighted undirected bipartite graph $H=\left(V_{H}, E_{H}\right)$ with vertices

$$
V_{H}=V \cup\{\{i, j\} \subseteq V: i \neq j\}
$$

and edges

$$
E_{H}=\left\{\{i,\{i, j\}\}: i \in V \backslash X, j \in V \backslash\{i\}, w(i, j)<s^{*}\right\} .
$$

We claim that $X \in P W S_{M M}\left(G^{\prime}\right)$ with a maximin score of $s^{*}=\frac{n}{2}$ in the corresponding completion if and only if there is a maximum-cardinality matching of size $|V \backslash X|$ in $H$.

$(\Rightarrow)$ Let $T=\left(V, w^{\prime \prime}\right)$ a completion of $G^{\prime}$ (and thereby of $G$ ) in which $X$ is the set of maximin winners with $s_{M M}(i, T)=s^{*}=\frac{n}{2}$ for all $i \in X$. For each $i \notin X$, there needs to be a $j \neq i$ with $w^{\prime \prime}(i, j)<s^{*}$. Collecting $\{i,\{i, j\}\}$ for each such pair gives a matching of size $|V \backslash X|$ in $H$ which is maximum since each vertex on one side of the bipartite graph is contained in it.

$(\Leftarrow)$ For the other direction, assume that there is a maximum matching of size $|V \backslash X|$. We construct a completion $T=\left(V, w^{\prime \prime}\right)$ of $G^{\prime}$ such that $X$ is the set of maximin winners. Note that every $i \in\left(V_{H} \cap V\right) \backslash X$ has to be contained in an edge $\{i,\{i, j\}\}$ in the matching. For each such edge, let $w^{\prime \prime}(i, j)=w^{\prime}(i, j)<s^{*}$ and $w^{\prime \prime}(j, i)=n-w^{\prime \prime}(i, j)$, implying that $s_{M M}(i, T)<s^{*}$. Otherwise, $T$ is an arbitrary completion of $G$.

Together, we have that $s_{M M}(i, T)=s^{*}$ for all $i \in X$ and $s_{M M}(i, T)<s^{*}$ for all $i \notin X$. Figure 10 illustrates the procedure for a 2-weighted tournament and the set $X=\{a\}$.

Case 3: $s^{*}<\frac{n}{2}$. For a given $s^{*}$, we construct an undirected unweighted bipartite graph $H^{s^{*}}=\left(V_{H}, E_{H}^{s^{*}}\right)$. Let $V_{H}$ be as before and

$$
E_{H}^{s^{*}}=\bigcup_{\substack{i \in X \\ j \neq i}}\left\{\{i,\{i, j\}\}: w(i, j) \leq s^{*} \leq n-w(j, i)\right\} \cup \bigcup_{\substack{i \in V \\ j \neq i}}\left\{\{i,\{i, j\}\}: w(i, j) \leq s^{*}-1\right\} .
$$

We claim that $X$ is in $P W S_{M M}(G)$ with a maximin score of $s^{*}$ in the corresponding completion if and only if there is a maximum-cardinality matching of size $|V|$ in $H^{s^{*}}$.

$(\Rightarrow)$ Let $T=\left(V, w^{\prime}\right)$ a completion of $G$ in which $X$ is the set of maximin winners with the maximum maximin score $s^{*}$. For every vertex $i \in V$, there has to be an $j \neq i$ such that $w^{\prime}(i, j)$ accounts for the maximin score of $i$. Also, since $s^{*}<\frac{n}{2}$, it cannot be the case that $j$ also derives its maximin score from $w^{\prime}(j, i)$. Therefore, the set of all such pairs $\{i,\{i, j\}\}$ is a valid matching of size $|V|$. It is obviously maximal.

$(\Leftarrow)$ For the other direction, assume that there is a maximum matching of size $|V|$. Note that every $i \in\left(V_{H} \cap V\right)$ is matched and define $j(i) \in V$ such that the edge $\{i,\{i, j(i)\}$ is contained in the matching. We construct a completion $T=\left(V, w^{\prime}\right)$ in which $X$ is the set of maximin winners. To this end, define

$$
\begin{aligned}
& w^{\prime}(i, j(i))=s^{*} \text { and } w^{\prime}(j(i), i)=n-s^{*} \text { for } i \in X, \text { and } \\
& w^{\prime}(i, j(i))=s^{*}-1 \text { and } w^{\prime}(j(i), i)=n-\left(s^{*}-1\right) \text { for } i \in V \backslash X .
\end{aligned}
$$

As long as there are unspecified edges $(i, j)$ in the completion, define

$$
\begin{gathered}
w^{\prime}(i, j)=\max \left\{w(i, j), s^{*}\right\} \text { and } w^{\prime}(j, i)=n-w(i, j) \text { if } i \in X, j \in V \text {, and } \\
w^{\prime}(i, j)=\max \left\{w(i, j), s^{*}-1\right\} \text { and } w^{\prime}(j, i)=n-w(i, j) \text { otherwise. }
\end{gathered}
$$


Note that $T$ is a proper completion of $G$. Now, we have $s_{M M}(i, T)=s^{*}$ for all $i \in X$ and $s_{M M}(i, T)<s^{*}$ for all $i \notin X$. This completes Case 3 .

It remains to be shown that only a limited number of possible $s^{*}$ (and thereby $H^{s^{*}}$ ) have to be considered. In contrast to the proof of Theorem 9 , we cannot employ a binary search method since there is no clear cut between a feasible and an infeasible integer interval. However, we can see that when $s^{*}$ is gradually incremented from 0 to $\frac{n}{2}-1$, whether an edge $\{i,\{i, j\}\}$ is contained in $E_{H}^{s^{*}}$ or not changes at most twice due to the definition of $E_{H}^{s^{*}}$. This partitions the integer interval $I=\left[0, \frac{n}{2}-1\right]$ of possible $s^{*}$ into a finite number of subintervals $I_{k}$ such that all $s^{*}$ within a single $I_{k}$ induce the same $H^{s^{*}}$. Therefore, it is sufficient to only consider one $s^{*}$ per $I_{k}$ and for this we choose the minimum. The set $S^{*}$ of possibly relevant target scores $s^{*}$ is then given by

$$
S^{*}=\bigcup_{k} \min I_{k} \subseteq \bigcup_{\substack{i \in X \\ j \neq i}}\{w(i, j), n-w(j, i)+1\} \cup \bigcup_{\substack{i \in V \\ j \neq i}}\{w(i, j)+1\} .
$$

The size of $S^{*}$ is obviously bounded by $3 n^{2}$.

All cases can be handled in polynomial time.

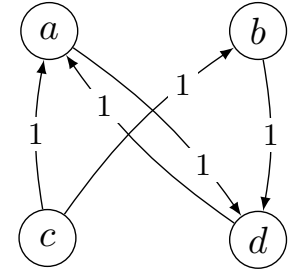

(i) A partial 2-weighted tournament $G$.

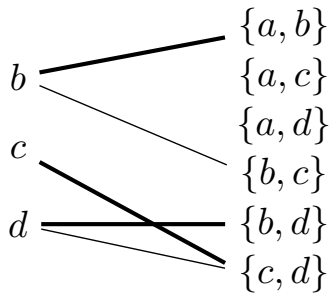

(iii) The constructed undirected bipartite graph $H$. Thick edges indicate a maximum-cardinality matching.

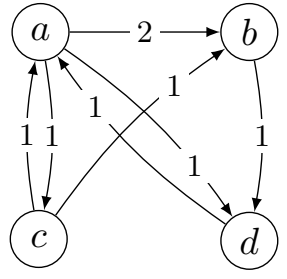

(ii) An extension $G^{\prime}$ reinforcing $\{a\}$.

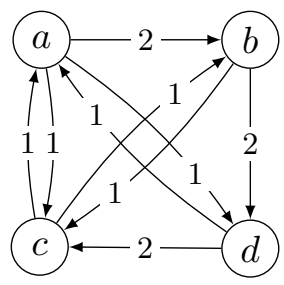

(iv) A completion of $G^{\prime}$ and $G$ with $M M\left(G^{\prime}\right)=\{a\}$

Figure 10: Illustration of the algorithm for checking whether a singleton $\{a\}$ is contained in $P W S_{M M}(G)$ for a partial 2-weighted tournament $G$. It is obvious that $a$ cannot have a maximin score of 2 in any completion or be the sole maximin winner with a maximin score of 0 . Therefore, we check the for the case $s^{*}=\frac{n}{2}=1$.

Lastly, we consider $\mathrm{NW}_{M M}$, for which we apply a similar technique as for $\mathrm{NW}_{B O}$ : to see whether $x \in N W_{M M}(G)$, we start from the graph $G^{x \leftarrow}$ and check whether some other alternative can achieve a higher maximin score than $x$ in a completion of $G^{x \leftarrow}$. 
Theorem 13. $\mathrm{NW}_{M M}$ can be solved in polynomial time.

Proof. We show how to check whether $x \in N W_{M M}(G)$ for a partial $n$-weighted tournament $G=(V, w)$. The maximin score of $x$ in $G^{x \leftarrow}$ is the worst case maximin score of $x$ among all proper completions of $G$.

For each $y \in V \backslash\{x\}$, the maximin score of $y$ in $G^{y \rightarrow}$ is the best possible maximin score of $y$ among the completions of $G$. If the maximin score of each $y$ in the corresponding $G^{y \rightarrow}$ is not more than the maximin score of $x$ in $G^{x \leftarrow}$, then $x \in N W_{M M}(G)$, otherwise $x \notin N W_{M M}(G)$.

\subsection{Ranked Pairs}

The method of ranked pairs $(R P)$ is the only resolute solution concept considered in this article. Given a weighted tournament $T=(V, w)$, it returns the unique undominated alternative of a transitive tournament $T^{\prime}$ on $V$ constructed in the following manner. First order the (directed) edges of $T$ in decreasing order of weight, breaking ties according to some exogenously given tie-breaking rule. Then start with an empty graph $T^{\prime}$ and consider the edges one by one according to this ordering. If the current edge can be added to $T^{\prime}$ without creating a cycle, then do so; otherwise discard the edge. ${ }^{11}$

As an example, consider the partial 5-weighted tournament depicted in Figure 11 $(i)$, which is a slightly modified version of the tournament considered in Figures 8 and 9. It is easy to see that $a$ is the ranked pairs winner in all completions of $G^{a \rightarrow}$, and likewise $b$ is the ranked pairs winner in all completions of $G^{b \rightarrow}$. On the other hand, there is no completion that has $c$ as a ranked pairs winner. Whether $d$ is a possible ranked pairs winner depends on the tie-breaking rule that is used, and in particular on how the tie-breaking rule ranks edges $(d, c)$ and $(b, d)$ : alternative $d$ is a possible ranked pairs winner if and only if $(d, c)$ is considered before $(b, d)$ (see Figure $11(i v)$ ). Since $R P$ is resolute, we have (assuming that the tie-breaking rule ranks $(d, c)$ over $(b, d))$

$$
\begin{aligned}
P W_{R P}(G) & =\{a, b, d\} \\
N W_{R P}(G) & =\emptyset \\
P W S_{R P}(G) & =\{\{a\},\{b\},\{d\}\} .
\end{aligned}
$$

It is readily appreciated that the winner determination problem for $R P$ is computationally tractable. The possible winner problem, on the other hand, turns out to be NP-hard. This also shows that tractability of the winner determination problem, while necessary for tractability of PW, is not generally sufficient.

Theorem 14. $\mathrm{PW}_{R P}$ is NP-complete.

Proof. We will work with an alternative characterization of ranked pairs winners that was introduced by Zavist and Tideman (1989). For a given a weighted tournament $T=(V, w)$

11. The variant of ranked pairs originally proposed by Tideman (1987), which was also used by Xia and Conitzer (2011), instead chooses a set of alternatives, containing any alternative that is selected by the above procedure for some way of breaking ties among edges with equal weight. We do not consider this irresolute version of ranked pairs because winner determination for this variant is NP-hard (Brill \& Fischer, 2012). As mentioned in Section 3, this immediately implies that all problems concerning possible or necessary winners are NP-hard as well. 


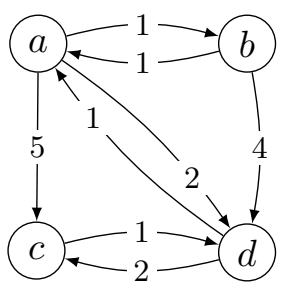

(i) A partial 5-weighted tournament $G$.

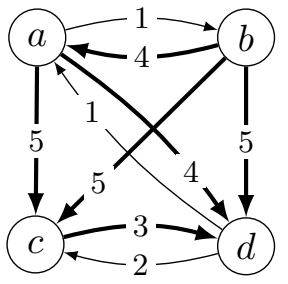

(iii) A completion of $G$ with ranked pairs winner $b$.

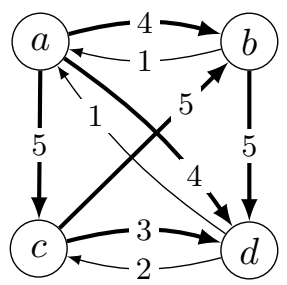

(ii) A completion of $G$ with ranked pairs winner $a$.

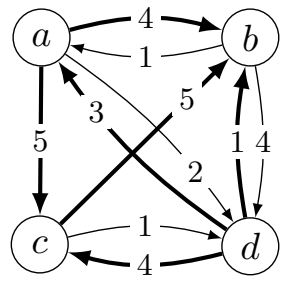

(iv) A completion of $G$ with ranked pairs winner $d$. Here, we assume that edge $(d, c)$ is considered before edge $(b, d)$.

Figure 11: Example of a 5-weighted partial tournament and completions relevant for possible ranked pairs winners. For each completion, the transitive tournament constructed by the ranked pairs procedure is indicated by the thick edges.

and a given tie-breaking rule, let $\succ$ denote the order in which edges are considered in the ranked pairs procedure. That is, $(x, y) \succ(u, v)$ if and only if either $w(x, y)>w(u, v)$ or $w(x, y)=w(u, v)$ and the tie-breaking rule ranks $(x, y)$ higher than $(u, v)$. Given a ranking $L$ of $V$, and two alternatives $a$ and $b$, we say that $a$ attains $b$ through $L$ if there exists a sequence of distinct alternatives $a_{1}, a_{2}, \ldots, a_{t}$, where $t \geq 2$, such that $a_{1}=a, a_{t}=b$, $a_{i} L a_{i+1}$, and

$$
\left(a_{i}, a_{i+1}\right) \succ(b, a) \text { for all } i \text { with } 1 \leq i<t .
$$

In this case, we will say that $a$ attains $b$ via $\left(a_{1}, a_{2}, \ldots, a_{t}\right)$. A ranking $L$ is called a stack if for any pair of alternatives $a$ and $b$ it holds that $a L b$ implies that $a$ attains $b$ through $L$. Zavist and Tideman (1989) have shown that an alternative is the ranked pairs winner if and only if it is the top element of a stack. ${ }^{12}$ Intuitively, the defining properties of a stack $L$ ensure that for all pairs $(a, b)$ of alternatives with $a L b$, by the point in time the edge $(b, a)$ is considered, it will be discarded because it would create a cycle.

Membership of $\mathrm{PW}_{R P}$ in NP is obvious, as for a given completion and a given tiebreaking rule, the ranked pairs winner can be found efficiently.

12. The characterization by Zavist and Tideman (1989) addresses the irresolute version of ranked pairs discussed in the previous footnote. Our adaptation to the resolute version of ranked pairs is a straightforward corollary. 


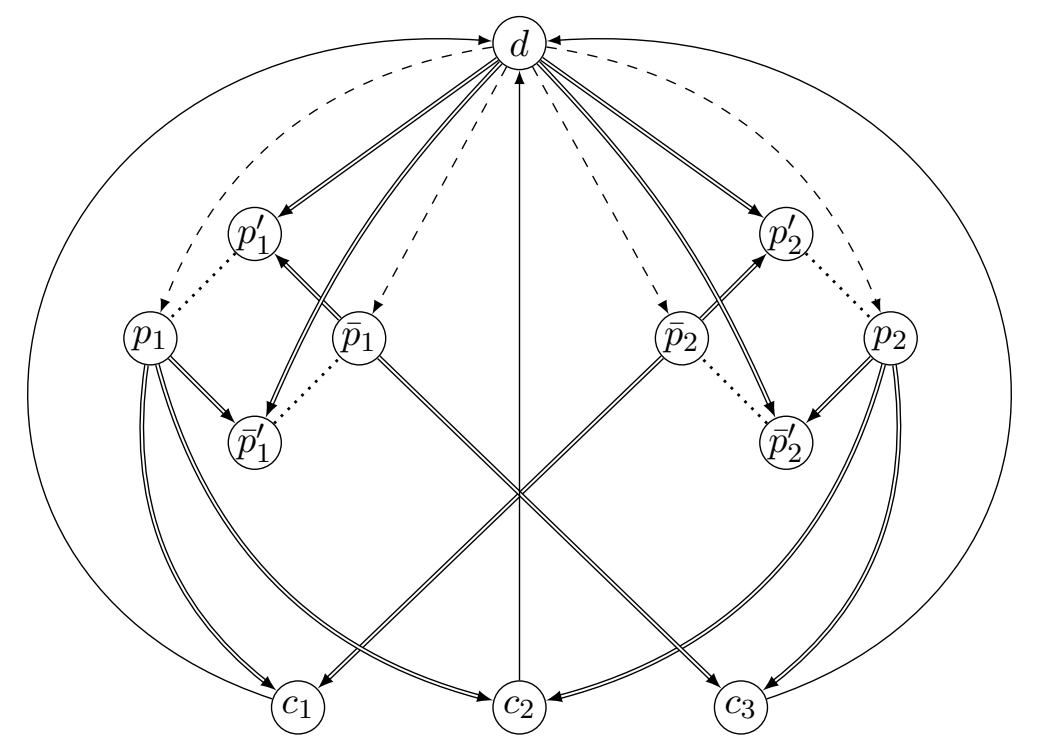

Figure 12: The partial 8-weighted tournament $G_{\varphi}$ for the Boolean formula $\varphi=\left\{p_{1}, \bar{p}_{2}\right\} \wedge$ $\left\{p_{1}, p_{2}\right\} \wedge\left\{\bar{p}_{1}, p_{2}\right\}$. Double-shafted arrows represent heavy edges, standard arrows represent medium edges, dashed arrows represent light edges, and dotted lines represent partial edges. For all pairs $(a, b)$ that are not connected by an arrow, we have $w_{\varphi}(x, y)=w_{\varphi}(y, x)=4$.

NP-hardness can be shown by a reduction from SAT. Our construction is based on the proof of Theorem 1 by Brill and Fischer (2012). For a Boolean formula $\varphi$ in conjunctive normal-form with a set $C$ of clauses and set $P$ of propositional variables, we construct a partial 8-weighted tournament $G_{\varphi}=\left(V_{\varphi}, w_{\varphi}\right)$ as follows. For each variable $p \in P, V_{\varphi}$ contains two literal alternatives $p$ and $\bar{p}$ and two auxiliary alternatives $p^{\prime}$ and $\bar{p}^{\prime}$. For each clause $c \in C$, there is an alternative $c$. Finally, there is an alternative $d$ for which membership in $P W_{R P}\left(G_{\varphi}\right)$ is to be decided.

In order to conveniently describe the weight function $w_{\varphi}$, let us introduce the following terminology. For two alternatives $x, y \in V_{\varphi}$, say that there is a heavy edge from $x$ to $y$ if $w_{\varphi}(x, y)=8$ (and therefore $w_{\varphi}(y, x)=0$ ). A medium edge from $x$ to $y$ means $w_{\varphi}(x, y)=6$ and $w_{\varphi}(y, x)=2$, and a light edge from $x$ to $y$ means $w_{\varphi}(x, y)=5$ and $w_{\varphi}(y, x)=3$. Finally, a partial edge between $x$ and $y$ means $w_{\varphi}(x, y)=w_{\varphi}(y, x)=1$.

We are now ready to define $w_{\varphi}$. For each variable $p \in P$, we have heavy edges from $p$ to $\bar{p}^{\prime}$ and from $\bar{p}$ to $p^{\prime}$, and partial edges between $p$ and $p^{\prime}$ and between $\bar{p}$ and $\bar{p}^{\prime}$. For each clause $c \in C$, we have a medium edge from $c$ to $d$ and a heavy edge from the literal alternative $\ell$ (with $\ell=p$ or $\ell=\bar{p}$ for some $p \in P$ ) to $c$ if the corresponding literal $\ell$ appears in clause $c$. Finally, we have heavy edges from $d$ to all auxiliary alternatives and light edges from $d$ to all literal alternatives. For all pairs $x, y$ for which no edge has been specified, we define $w_{\varphi}(x, y)=w_{\varphi}(y, x)=4$. An example is shown in Figure 12. Observe that the only pairs of alternatives for which $w_{\varphi}$ is not fully specified are those pairs that are connected by a partial edge. 


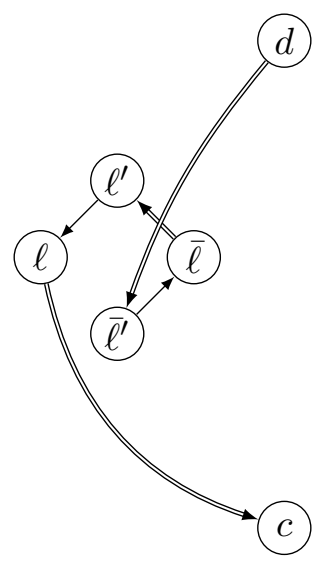

(i) $S_{c}=\left(d, \bar{\ell}^{\prime}, \bar{\ell}, \ell^{\prime}, \ell, c\right)$

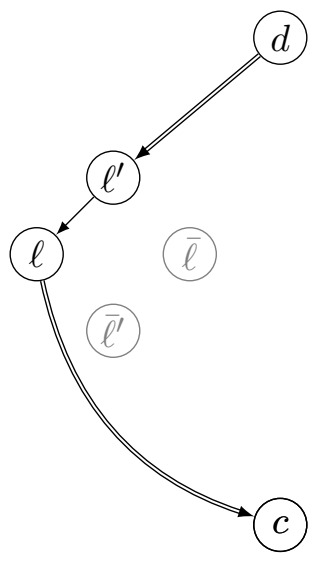

(ii) $S_{c}=\left(d, \ell^{\prime}, \ell, c\right)$

Figure 13: Two possibilities for the sequence $S_{c}$.

We will now show that alternative $d$ is a possible ranked pairs winner in $G_{\varphi}$ if and only if $\varphi$ is satisfiable. Intuitively, choosing a completion such that $\left(p^{\prime}, p\right) \succ\left(\bar{p}^{\prime}, \bar{p}\right)$ corresponds to setting the variable $p$ to true.

$(\Rightarrow)$ First assume that $d \in P W_{R P}$ and let $T \in\left[G_{\varphi}\right]$ be a completion of $G_{\varphi}$ with $R P(T)=\{d\}$. Consider a stack $L$ with top element $d$ and an alternative $c$ corresponding to a clause in $\varphi$. Since $L$ is a stack and $d L c, d$ attains $c$ though $L$ via some sequence $S_{c}$. (If $d$ attains $c$ via several sequences, fix one of them arbitrarily.) Since $w_{\varphi}(c, d)=6$, all edges in the sequence $S_{c}$ must be heavy, medium, or appropriate completions of partial edges. Therefore, $S_{c}$ must have one of the following two forms (depicted in Figure 13):

$$
S_{c}=\left(d, \bar{\ell}^{\prime}, \bar{\ell}, \ell^{\prime}, \ell, c\right) \quad \text { or } \quad S_{c}=\left(d, \ell^{\prime}, \ell, c\right),
$$

where $\ell$ is some literal. The former is in fact not possible because $w_{\varphi}\left(\ell, \bar{\ell}^{\prime}\right)=8$ implies that $\bar{\ell}^{\prime}$ does not attain $\ell$ through $L$. Therefore, each $S_{c}$ is of the form $S_{c}=\left(d, \ell^{\prime}, \ell, c\right)$ for some literal $\ell$.

Now define assignment $\alpha$ by setting to true all literals that are contained in one of the sequences $S_{c}, c \in C$. We claim that $\alpha$ is a satisfying assignment for $\varphi$.

In order to show that $\alpha$ is well-defined, suppose there exists a literal $\ell$ such that both $\ell$ and $\bar{\ell}$ are set to true under $\alpha$. This implies that there exist $c_{1}$ and $c_{2}$ such that $d$ attains $c_{1}$ via $S_{c_{1}}=\left(d, \ell^{\prime}, \ell, c_{1}\right)$ and $d$ attains $c_{2}$ via $S_{c_{2}}=\left(d, \bar{\ell}^{\prime}, \bar{\ell}, c_{2}\right)$. In particular, $\ell^{\prime} L \ell$ and $\bar{\ell}^{\prime} L \bar{\ell}$. However, it is easily verified that any stack ranks $\ell$ higher than $\bar{\ell}^{\prime}$ (because $w_{\varphi}\left(\ell, \bar{\ell}^{\prime}\right)=8$ ) and $\bar{\ell}$ higher than $\ell^{\prime}$ (because $w_{\varphi}\left(\bar{\ell}, \ell^{\prime}\right)=8$ ). Thus, there is an $L$-cycle $\ell L \bar{\ell}^{\prime} L \bar{\ell} L \ell^{\prime} L \ell$, contradicting the assumption that $L$ is a stack.

In order to show that $\alpha$ satisfies $\varphi$, consider an arbitrary clause $c$. As $d$ attains $c$ via $S_{c}=\left(d, \ell^{\prime}, \ell, c\right)$ and $w_{\varphi}(c, d)=6$, we have that $w_{\varphi}\left(\ell, y_{j}\right) \geq 6$. By definition of $w_{\varphi}(\cdot, \cdot)$, this implies that literal $\ell$ appears in clause $c$. Furthermore, $\ell$ is set to true under $\alpha$ because $\ell$ is contained in $S_{c}$.

$(\Leftarrow)$ For the other direction, assume that $\varphi$ is satisfiable and let $\alpha$ be a satisfying assignment. We use $\alpha$ to construct a completion $T=\left(V_{\varphi}, w_{\alpha}\right) \in\left[G_{\varphi}\right]$ with $R P(T)=\{d\}$. 
On partial edges, the weight function $w_{\alpha}$ is defined as follows. If literal $\ell$ is set to true under $\alpha$, let $w_{\alpha}\left(\ell^{\prime}, \ell\right)=7$ and $w_{\alpha}\left(\ell, \ell^{\prime}\right)=1$. Otherwise, let $w_{\alpha}\left(\ell^{\prime}, \ell\right)=1$ and $w_{\alpha}\left(\ell, \ell^{\prime}\right)=7$.

We now show that $R P(T)=\{d\}$ by going through the procedure that constructs the transitive tournament $T^{\prime}$, starting with the empty tournament on $V_{\varphi} \cdot{ }^{13}$ First, the set of all edges with weight 7 or more will be added, because there are no cycles among those edges. This set consists of the heavy edges and the (previously) partial edges. Next, the medium edges are considered. All these edges are of the form $(c, d)$ where $c$ is an alternative corresponding to a clause. Since $\alpha$ is a satisfying assignment, $T^{\prime}$ already contains paths from $d$ to every clause alternative $c$. Therefore, all of the edges $(c, d)$ with $c \in C$ will be discarded. In the next step, all light edges (i.e., edges of weight 5) are considered. All of those edges are of the form $(d, \ell)$ for a literal $\ell$. Therefore, all of those edges can be added to $T^{\prime}$ without creating a cycle ( $d$ has no ingoing edges in $T^{\prime}$ ). After adding the light edges, $d$ has an outgoing edge to all literal alternatives $\ell$ and to all auxiliary alternatives $\ell^{\prime}$. Furthermore, all edges from a clause candidate $c$ to $d$ have already been discarded. Thus, $d$ is the unique undominated alternative in $T^{\prime}$, i.e., $R P(T)=\{d\}$.

Since the ranked pairs method is resolute, hardness of $\mathrm{PWS}_{R P}$ follows immediately.

Corollary 2. $\mathrm{PWS}_{R P}$ is NP-complete.

Computing necessary ranked pairs winners turns out to be coNP-complete. This is again somewhat surprising, as computing necessary winners is often considerably easier than computing possible winners, for both partial tournaments and partial preference profiles (Xia \& Conitzer, 2011).

Theorem 15. $\mathrm{NW}_{R P}$ is coNP-complete.

Proof. Membership in coNP is again obvious. For hardness, we give a reduction from UNSAT that is a slight variation of the reduction in the proof of Theorem 14. Let $G_{\varphi}^{\prime}$ be the partial 8-weighted tournament that results form $G_{\varphi}$ by adding a new alternative $d^{*}$ which has heavy edges to all alternatives in $V_{\varphi}$ except $d$. Furthermore, there is a light edge from $d$ to $d^{*}$. We show that $d^{*}$ is a necessary ranked pairs winner in $G_{\varphi}^{\prime}$ if and only if $\varphi$ is unsatisfiable.

$(\Rightarrow)$ Assume for contradiction that $N W_{R P}\left(G_{\varphi}^{\prime}\right)=\left\{d^{*}\right\}$ and $\varphi$ is satisfiable. Let $\alpha$ be a satisfying assignment and define the tournament $T=\left(V_{\varphi} \cup\left\{d^{*}\right\}, w_{\alpha}^{\prime}\right) \in\left[G_{\varphi}^{\prime}\right]$ such that $w_{\alpha}^{\prime}$ coincides with $w_{\alpha}$ (as defined in the proof of Theorem 14) for all partial edges. By the same arguments as in the proof of Theorem 14, it follows that $d$ does not have any ingoing edges in the tournament $T^{\prime}$ constructed by the ranked pairs procedure. At the point in time when the edge $\left(d, d^{*}\right)$ is considered, it will be added to $T^{\prime}$. This yields $R P(T)=\{d\}$, contradicting the assumption that $N W_{R P}\left(G_{\varphi}^{\prime}\right)=\left\{d^{*}\right\}$.

$(\Leftarrow)$ Assume for contradiction that $\varphi$ is unsatisfiable and there exists a completion $T \in\left[G_{\varphi}^{\prime}\right]$ with $R P(T)=\{x\} \neq\left\{d^{*}\right\}$. It follows that $x=d$. (All alternatives in $V_{\varphi} \backslash\{d\}$ have an incoming heavy edge (from $d^{*}$ ), and all heavy edges will be added because there is no cycle among them.) By the same argument as in the proof of Theorem 14, it follows that $\varphi$ is satisfiable, contradicting our assumption.

13. The following arguments are independent of the choice of a particular tie-breaking rule. 


\section{Possible Winning Subsets}

We considered the problem whether a subset of alternatives is a possible winning set (PWS). In addition, it may be of interest whether a subset of alternatives is among the winners in some completion, i.e., whether there is a completion for which all the alternatives in the subset (and possibly other alternatives) are in the choice set. We will refer to the latter problem as PWSS (possible winning subset). We note that an oracle to solve PWSS can be used to solve PW. If we want to check whether $i \in P W(G)$, we simply check whether $\{i\} \in P W S S(G)$. We are not aware of any direct algorithmic relation between the problems PWS and PWSS.

We examined the computational complexity of $\mathrm{PWSS}_{S}$ for most of the solution concepts considered in this article. Since the arguments are often very similar to proofs already given, we briefly summarize our findings here.

$C O N D$ As there is never more than one Condorcet winner, every $X \in P W S S_{C O N D}(G)$ is a singleton and the problem reduces to computing $P W_{C O N D}(G)$.

$C N L$ For $\mathrm{PWSS}_{C N L}$, note that for a nonempty set $X \subseteq V, X \notin P W S S_{C N L}(G)$ if and only if $|V|>1$ and every completion of $G^{-1}$ has a Condorcet winner which is furthermore located in $X$. Therefore,

$$
X \in P W S S_{C N L}(G) \text { if and only if } \begin{cases}|V|=1 & \text { or } \\ \emptyset \in P W S_{C O N D}\left(G^{-1}\right) & \text { or } \\ P W_{C O N D}\left(G^{-1}\right) \backslash X \neq \emptyset . & \end{cases}
$$

$C O$ Just as for the other problems, polynomial computability of $\mathrm{PWSS}_{C O}$ follows from the corresponding result for $\mathrm{PWSS}_{B O}$.

$T C$ The problem $\mathrm{PWSS}_{T C}$ can be solved in polynomial time. In fact, it can be shown that for a partial tournament $G$ and a set of alternatives $X$, it is sufficient to check whether $X \subseteq P W_{T C}(G)$ (with an additional argument if $|X|=2$ ) in order to determine whether $X \in P W S S_{T C}(G)$.

$B O$ The argument and algorithm for checking whether $X \in P W S S_{B O}(G)$ is almost the same as the argument for $\mathrm{PWS}_{B O}$ in Theorem 9. The only difference is that $s_{B O}(v, T)$ may now be up to $s^{*}$ instead of $s^{*}-1$ for $v \in V \backslash X$ in $T \in[G]$. Consequently, we only need to redefine $b_{s^{*}}(v)$ to $s^{*}-s_{B O}(v, G)$ for all $v \in V \backslash X$.

$M M$ The proof for efficient computability of checking whether $X \in P W S_{M M}(G)$ can be modified to accommodate $\mathrm{PWSS}_{M M}$. More precisely, the second basic assumption is now $w(i, j) \leq s^{*}$ for $i \notin X, j \in V$. For $s^{*}=\frac{n}{2}$, it is sufficient to check whether $G^{\prime}$ is an extension of $G$. For $s^{*}<\frac{n}{2}$, edges $\{i,\{i, j\}\}$ with $i \in X$ are now contained in $E_{H}^{s^{*}}$ if $w(i, j) \leq s^{*}$. The rest of the argument can be adjusted appropriately. For $s^{*}>\frac{n}{2}$, nothing changes.

$R P$ Since $\mathrm{PW}_{R P}$ is NP-complete (Theorem 14), we get NP-hardness of PWSS $R P$ by the oracle argument above. Since membership in NP is obvious, the problem is NP-complete. 
The complexity of PWSS $\mathrm{PC}_{U}$ is left open. Minor modification of our hardness construction for $\mathrm{PWS}_{U C}$ will not do the trick. In that argument, the crucial question was whether there is a completion that excludes certain alternatives from the choice set. This does not help for $\mathrm{PWSS}_{U C}$.

\section{Discussion}

The problem of computing possible and necessary winners for partial preference profiles has recently received a lot of attention. In this article, we have investigated this problem in a setting where partially specified (weighted or unweighted) tournaments instead of profiles are given as input. We have summarized our findings in Table 1.

A key conclusion is that computational problems for partial tournaments can be significantly easier than their counterparts for partial profiles. For example, possible Borda or maximin winners can be found efficiently for partial tournaments, whereas the corresponding problems for partial profiles are NP-complete (Xia \& Conitzer, 2011). Furthermore, computing possible and necessary Copeland winners is NP-hard and coNP-hard respectively for partial preference profiles (Xia \& Conitzer, 2011). In contrast, we showed that even $\mathrm{PWS}_{C O}$ can be solved in polynomial time for partial tournaments. As for negative (hardness) results, they can be tempered by the fact that when some parameters of the problem are bounded by a constant, some of these hard problems may be solved in polynomial time. In particular, Yang and Guo (2013) have shown that PWS ${ }_{U C}$ is polynomial-time solvable if the size of the given subset $X$ is bounded by a constant. ${ }^{14}$

While tractability of the winner determination problem is necessary for tractability of the possible or necessary winners problems, the results for ranked pairs in Section 5.3 show that it is not sufficient. We further considered the problem of deciding whether a given subset of alternatives equals the winning set for some completion of the partial tournament. The results for the uncovered set in Section 4.4 imply that this problem cannot be reduced in polynomial time to the computation of possible or necessary winners; whether a reduction exists in the opposite direction remains an open problem.

Partial tournaments have also been studied in their own right, independent of their possible completions. For instance, Peris and Subiza (1999) and Dutta and Laslier (1999) have generalized several solution concepts on tournaments to partial tournaments. The common point with the approach we follow here is the nature of the input, namely, partial tournaments. However, Peris and Subiza (1999) and Dutta and Laslier (1999) define solution concepts for partial tournaments by directly generalizing the usual definition on tournaments. This is in contrast to our definitions, which are based on the completions of the input partial tournament. The notion of possible winners suggests a canonical way to generalize any solution concept defined on tournaments to partial tournaments. This way of extending tournament solutions to partial tournaments is referred to as the "conservative extension" and inherits various axiomatic properties which the original tournament solutions satisfies for tournaments (Brandt et al., 2014). The positive computational results in this article are an indication that this may be a promising approach.

14. Yang and Guo (2013) also give hardness and fixed-parameter tractability results for a generalization of the Banks set to partial tournaments. 
We also highlight another way of viewing algorithmic results concerning possible and necessary winners. There is a burgeoning literature in computational social choice which deals with the problem of manipulation and control in voting (Bartholdi, III, Tovey, \& Trick, 1989, 1992; Faliszewski \& Procaccia, 2010). If a given alternative is already a necessary winner, then there is no need to invest effort into influencing the remaining comparisons or votes to make it winning. Moreover, our results also have implications on a partial tournament version of the coalitional manipulation problem: coalitional tournament manipulation, in its constructive version, is defined as follows. Given a partial tournament $(V, E)$, a subset $X \subseteq V$, and a distinguished alternative $x$, is there a way to complete the missing edges in $X \times X$ such that $x$ is a winner? Informally, do the players in $X$ have a way of fixing the winners of the matches among themselves so as to make $x$ win?

Constructive coalitional tournament manipulation is polynomial-time solvable whenever PW is. Likewise, the destructive version of coalitional tournament manipulation (is there a way to complete the edges within $X$ such that candidate $x$ is not winning?) is polynomial whenever NW is.

Regarding future work, we have not yet examined the complexity of computing possible and necessary winners for some attractive tournament solutions such as the minimal covering set and weighted versions of the top cycle and the uncovered set (De Donder, Le Breton, \& Truchon, 2000). ${ }^{15}$

An interesting related question that goes beyond the computation of possible and necessary winners is the following: when the winners are not yet fully determined, which unknown comparisons need to be learned, which pairs of candidates do we have to compare, or which matches should be played? This problem can be seen as a tournament-based version of the preference elicitation problem (Conitzer \& Sandholm, 2002; Ding \& Lin, 2013; Walsh, 2008). While the standard version of the problem looks for minimal sets of queries to voters about pairwise preferences between candidates, in the tournament version a query bears on a pair of candidates and its output is an edge between these two candidates, in one direction or the other. Procaccia (2008) considers a similar question for COND. The construction of a policy tree defining an optimal protocol minimizing the number of questions to be asked or the number of matches to be played, in the worst case or on average, is an even more challenging issue that we leave for future research.

\section{Acknowledgments}

Previous versions of this paper have been presented at the 11th International Conference on Autonomous Agents and Multi-Agent Systems (AAMAS 2012) and at the 4th International Workshop on Computational Social Choice (COMSOC 2012). We are grateful to Felix Brandt for extensive discussions and useful advice. We also thank Gerhard Woeginger for hints towards improving our previous pseudo-polynomial time algorithms for $\mathrm{PWS}_{B O}$ and $\mathrm{PWS}_{M M}$ and various anonymous reviewers, whose comments greatly helped us to improve our paper. This material is based on work supported by the Deutsche Forschungsgemeinschaft under grants BR 2312/9-1, BR 2312/10-1, and FI 1664/1-1. Haris Aziz is supported by the Australian Government as represented by the Department of Broadband, Commu-

15. Brill, Freeman, and Conitzer (2016) have recently shown that computing possible and necessary winners for the bipartisan set (Laffond, Laslier, \& Le Breton, 1993) is intractable. 
nications and the Digital Economy and the Australian Research Council through the ICT Centre of Excellence program. Markus Brill has been supported by a Feodor Lynen research fellowship of the Alexander von Humboldt Foundation and by the ERC under Starting Grant 639945 ("ACCORD"). Jérôme Lang has been supported by the ANR project CoCoRICoCoDec. Paul Harrenstein has been supported by the ERC under Advanced Grant 291528 ("RACE").

\section{References}

Aziz, H., Gaspers, S., Mackenzie, S., Mattei, N., Stursberg, P., \& Walsh, T. (2014). Fixing a balanced knockout tournament. In Proceedings of the 28th AAAI Conference on Artificial Intelligence (pp. 552-558). AAAI Press.

Aziz, H., Walsh, T., \& Xia, L. (2015). Possible and necessary allocations via sequential mechanisms. In Proceedings of the 23rd International Joint Conference on Artificial Intelligence (pp. 468-474).

Bachrach, Y., Betzler, N., \& Faliszewski, P. (2010). Probabilistic possible winner determination. In Proceedings of the 24th AAAI Conference on Artificial Intelligence (pp. 697-702). AAAI Press.

Bartholdi, III, J., Tovey, C. A., \& Trick, M. A. (1989). The computational difficulty of manipulating an election. Social Choice and Welfare, 6(3), 227-241.

Bartholdi, III, J., Tovey, C. A., \& Trick, M. A. (1992). How hard is it to control an election? Mathematical and Computer Modelling, 16(8-9), 27-40.

Baumeister, D., Faliszewski, P., Lang, J., \& Rothe, J. (2012). Campaigns for lazy voters: truncated ballots. In Proceedings of the 11th International Conference on Autonomous Agents and Multi-Agent Systems (pp. 577-584). IFAAMAS.

Baumeister, D., \& Rothe, J. (2010). Taking the final step to a full dichotomy of the possible winner problem in pure scoring rules. In Proceedings of the 19th European Conference on Artificial Intelligence (pp. 1019-1020).

Betzler, N., \& Dorn, B. (2010). Towards a dichotomy for the possible winner problem in elections based on scoring rules. Journal of Computer and System Sciences, 76(8), 812-836.

Betzler, N., Hemmann, S., \& Niedermeier, R. (2009). A multivariate complexity analysis of determining possible winners given incomplete votes. In Proceedings of the 21st International Joint Conference on Artificial Intelligence (pp. 53-58). AAAI Press.

Brandt, F., Brill, M., \& Harrenstein, P. (2014). Extending tournament solutions. In Proceedings of the 28th AAAI Conference on Artificial Intelligence (pp. 580-586). AAAI Press.

Brandt, F., Brill, M., \& Harrenstein, P. (2016). Tournament solutions. In F. Brandt, V. Conitzer, U. Endriss, J. Lang, \& A. D. Procaccia (Eds.), Handbook of Computational Social Choice (chap. 3). Cambridge University Press. (Forthcoming) 
Brill, M., \& Fischer, F. (2012). The price of neutrality for the ranked pairs method. In Proceedings of the 26th AAAI Conference on Artificial Intelligence (pp. 1299-1305). AAAI Press.

Brill, M., Freeman, R., \& Conitzer, V. (2016). Computing possible and necessary equilibrium actions (and bipartisan set winners). In Proceedings of the 30th AAAI Conference on Artificial Intelligence. AAAI Press. (Forthcoming)

Chevaleyre, Y., Lang, J., Maudet, N., \& Monnot, J. (2011). Compilation and communication protocols for voting rules with a dynamic set of candidates. In Proceedings of the $13 h$ Conference on Theoretical Aspects of Rationality and Knowledge (pp. 153-160).

Chevaleyre, Y., Lang, J., Maudet, N., Monnot, J., \& Xia, L. (2012). New candidates welcome! Possible winners with respect to the addition of new candidates. Mathematical Social Sciences, 64(1), 74-88.

Conitzer, V., \& Sandholm, T. (2002). Vote elicitation: Complexity and strategy-proofness. In Proceedings of the 18th National Conference on Artificial Intelligence (pp. 392397). AAAI Press.

Cook, W. J., Cunningham, W. H., Pulleyblank, W. R., \& Schrijver, A. (1998). Combinatorial optimization. Wiley and Sons.

De Donder, P., Le Breton, M., \& Truchon, M. (2000). Choosing from a weighted tournament. Mathematical Social Sciences, 40(1), 85-109.

Ding, N., \& Lin, F. (2013). Voting with partial information: what questions to ask? In Proceedings of the 12th International Conference on Autonomous Agents and MultiAgent Systems (pp. 1237-1238). IFAAMAS.

Dutta, B., \& Laslier, J.-F. (1999). Comparison functions and choice correspondences. Social Choice and Welfare, 16(4), 513-532.

Edmonds, J. (1965). Paths, trees and flowers. Canadian Journal of Mathematics, 17, 449-467.

Faliszewski, P., \& Procaccia, A. D. (2010). AI's war on manipulation: Are we winning? AI Magazine, 31(4), 53-64.

Filmus, Y., \& Oren, J. (2014). Efficient voting via the top- $k$ elicitation scheme: a probabilistic approach. In Proceedings of the 15th ACM Conference on Economics and Computation (pp. 295-312). ACM Press.

Good, I. J. (1971). A note on Condorcet sets. Public Choice, 10(1), 97-101.

Hazon, N., Aumann, Y., Kraus, S., \& Wooldridge, M. (2012). On the evaluation of election outcomes under uncertainty. Artificial Intelligence, 189, 1-18.

Kalech, M., Kraus, S., Kaminka, G. A., \& Goldman, C. V. (2011). Practical voting rules with partial information. Journal of Autonomous Agents and Multi-Agent Systems, 22(1), 151-182. 
Kern, W., \& Paulusma, D. (2004). The computational complexity of the elimination problem in generalized sports competitions. Discrete Optimization, 1(2), 205-214.

Konczak, K., \& Lang, J. (2005). Voting procedures with incomplete preferences. In Proceedings of the Multidisciplinary Workshop on Advances in Preference Handling (pp. 124-129).

Laffond, G., Laslier, J.-F., \& Le Breton, M. (1993). The bipartisan set of a tournament game. Games and Economic Behavior, 5(1), 182-201.

Lang, J., Pini, M. S., Rossi, F., Salvagnin, D., Venable, K. B., \& Walsh, T. (2012). Winner determination in voting trees with incomplete preferences and weighted votes. Journal of Autonomous Agents and Multi-Agent Systems, 25(1), 130-157.

Laslier, J.-F. (1997). Tournament solutions and majority voting. Springer-Verlag.

Lu, T., \& Boutilier, C. (2011). Vote elicitation with probabilistic preference models: Empirical estimation and cost tradeoffs. In Proceedings of the 2nd International Conference on Algorithmic Decision Theory (pp. 135-149). Springer-Verlag.

Lu, T., \& Boutilier, C. (2013). Multiwinner social choice with incomplete preferences. In Proceedings of the 23rd International Joint Conference on Artificial Intelligence (pp. 263-270). AAAI Press.

Oren, J., Filmus, Y., \& Boutilier, C. (2013). Efficient vote elicitation under candidate uncertainty. In Proceedings of the 23rd International Joint Conference on Artificial Intelligence (pp. 309-316). AAAI Press.

Peris, J. E., \& Subiza, B. (1999). Condorcet choice correspondences for weak tournaments. Social Choice and Welfare, 16(2), 217-231.

Pini, M. S., Rossi, F., Venable, K. B., \& Walsh, T. (2011). Possible and necessary winners in voting trees: Majority graphs vs. profiles. In Proceedings of the 10th International Conference on Autonomous Agents and Multi-Agent Systems (pp. 311-318). IFAAMAS.

Procaccia, A. (2008). A note on the query complexity of the Condorcet winner. Information Processing Letters, 108(6), 390-393.

Rastegari, B., Condon, A., Immorlica, N., \& Leyton-Brown, K. (2013). Two-sided matching with partial information. In Proceedings of the 14th ACM Conference on Electronic Commerce (pp. 733-750). ACM Press.

Schrijver, A. (2003). Combinatorial optimization-polyhedra and efficiency. Springer.

Schwartz, B. L. (1966). Possible winners in partially completed tournaments. SIAM Review, 8(3), 302-308.

Schwartz, T. (1986). The logic of collective choice. Columbia University Press.

Smith, J. H. (1973). Aggregation of preferences with variable electorate. Econometrica, 41(6), 1027-1041. 
Tideman, T. N. (1987). Independence of clones as a criterion for voting rules. Social Choice and Welfare, 4(3), 185-206.

Vu, T., Altman, A., \& Shoham, Y. (2009). On the complexity of schedule control problems for knockout tournaments. In Proceedings of the 8th International Conference on Autonomous Agents and Multi-Agent Systems (pp. 225-232). IFAAMAS.

Walsh, T. (2007). Uncertainty in preference elicitation and aggregation. In Proceedings of the 22nd AAAI Conference on Artificial Intelligence (pp. 3-8). AAAI Press.

Walsh, T. (2008). Complexity of terminating preference elicitation. In Proceedings of the 7th International Conference on Autonomous Agents and Multi-Agent Systems (pp. 967-974). IFAAMAS.

Xia, L., \& Conitzer, V. (2011). Determining possible and necessary winners under common voting rules given partial orders. Journal of Artificial Intelligence Research, 41, 2567.

Yang, Y., \& Guo, J. (2013). Possible winner problems on partial tournaments: A parameterized study. In Proceedings of the 3rd International Conference on Algorithmic Decision Theory (Vol. 8176, pp. 425-439). Springer-Verlag.

Zavist, T. M., \& Tideman, T. N. (1989). Complete independence of clones in the ranked pairs rule. Social Choice and Welfare, 6(2), 167-173. 